\title{
THE
}

UNIVERSITY

University of Rhode Island

OF RHODE ISLAND

DigitalCommons@URI

8-3-1964

\section{Linear Magnetic Chains with Anisotropic Coupling}

Jill C. Bonner

University of Rhode Island

Michael E. Fisher

Follow this and additional works at: https://digitalcommons.uri.edu/phys_facpubs

Terms of Use

All rights reserved under copyright.

\section{Citation/Publisher Attribution}

Bonner, Jill C. and Michael Fisher. (1964). Linear Magnetic Chains with Anisotropic Coupling. Phys. Rev. 135, A640. doi: 10.1103/PhysRev.135.A640

Available: http://dx.doi.org/10.1103/PhysRev.135.A640

This Article is brought to you for free and open access by the Physics at DigitalCommons@URI. It has been accepted for inclusion in Physics Faculty Publications by an authorized administrator of DigitalCommons@URI. For more information, please contact digitalcommons-group@uri.edu. 


\title{
Linear Magnetic Chains with Anisotropic Coupling
}

\author{
JILL C. BONNER \\ Department of Physics, Royal Holloway College, Englefield Green, Surrey, England \\ AND
}

Michael E. Fisher

Wheatstone Physics Laboratory, King's College, London W. C. 2, England

(Received 11 February 1964; revised manuscript received 8 April 1964)

Linear chains (and rings) of $S=\frac{1}{2}$ spins with the anisotropic (Ising-Heisenberg) Hamiltonian

$$
\mathcal{H C}=-2 J \sum_{i=1}^{N}\left\{S_{i}^{z} S_{i+1}^{z}+\gamma\left(S_{i}^{x} S_{i+1}^{x}+S_{i}^{y} S_{i+1}^{y}\right)\right\}-g \beta \sum_{i=1}^{N} \mathbf{H} \cdot \mathbf{S}_{i}
$$

have been studied by exact machine calculations for $N=2$ to $11, \gamma=0$ to 1 and for ferro- and antiferromagnetic coupling. The results reveal the dependence on finite size and anisotropy of the spectrum and dispersion laws, of the energy, entropy, and specific heat, of the magnetization and susceptibilities, and of the pair correlations. The limiting $N \rightarrow \infty$ behavior is accurately indicated, for all $\gamma$, in the region $k T /|J| \geqslant 0.5$ which includes the maxima in the specific heat and susceptibility. The behavior of thermal and magnetic properties of infinite chains at lower temperatures is estimated by extrapolation. For infinite antiferromagnetic chains the ground-state degeneracy, the anisotropy gap, and the magnetization, perpendicular susceptibility, and pair correlations at $T=0$ are similarly studied. Estimates of the long-range order suggest that it vanishes only at the Heisenberg limit $\gamma=1$ and confirm the accuracy of Walker's perturbation series in $\gamma$.

\section{INTRODUCTION}

$\mathrm{E}^{\mathrm{x}}$ XPERIMENTAL and theoretical studies have shown that in many magnetic materials, the magnetic part of the Hamiltonian may be quite accurately represented as a set of localized spins $\mathbf{S}_{i}$ with bilinear interactions. For certain highly anisotropic systems, ${ }^{1}$ the coupling energy can be approximated by the pure Ising form $-2 J S_{i}{ }^{z} S_{j}$, but for most systems the anisotropy, although important, is not very large and the pure Heisenberg coupling $-2 J \mathbf{S}_{i} \cdot \mathbf{S}_{j}$ is more realistic. Between these extremes is the anisotropic "intermediate" coupling $-2 J\left\{\gamma \mathbf{S}_{i} \cdot \mathbf{S}_{j}+(1-\gamma) S_{i}{ }^{z} S_{j}{ }^{z}\right\}$ which reduces to the Ising case when $\gamma=0$ and the Heisenberg case when $\gamma=1$.

With pure Ising interaction the partition function of a finite or infinite chain of spins in a parallel magnetic field, and thence the thermal and magnetic properties, may be calculated exactly. ${ }^{2}$ For simple two-dimensional Ising lattices exact solutions may be obtained in zero field. ${ }^{2}$ For the Heisenberg, or intermediate $\gamma$ spin Hamiltonians, however, exact closed formulas for the finite-temperature behavior have not been found even for the linear chain despite much theoretical effort (see below). Although, naturally, greatest interest attaches to three-dimensional lattices, the properties of linear chains with non-Ising spin coupling are of both experimental and theoretical significance.

${ }^{1}$ A. H. Cooke, D. T. Edmonds, C. B. P. Flinn, and W. P. Wolf Proc. Phys. Soc. (London) 74, 791 (1959); M. Ball, M. T. Hutchings, M. J. M. Leask, and W. P. Wolf, Proceedings of the Eighth International Congress on Low-Temperature Physics (to be published); M. Ball, M. J. M. Leask, W. P. Wolf, and A. F. G. Wyatt, J. Appl. Phys. 34, 1104 (1963).

${ }^{2}$ C. Domb, Advan. Phys. 9, 149 (1960). The perpendicular susceptibility (in zero field) may also be calculated exactly with Ising coupling in one and two dimensions. See Refs. 43 and 44.
Experimentally a number of crystals are known, ${ }^{3}$ for example copper tetramine sulfate monohydrate, ${ }^{3 \mathrm{a}, \mathrm{b}, 4}$ in which the magnetic ions are arranged in chains with strong interactions within each chain but rather weak interactions between chains. Except at the lowest temperatures, the chains should be almost independent and theoretical values based on a one-dimensional model may be confronted directly with experimental measurements. Furthermore, the experimental evidence on various cupric quinone complex salts ${ }^{3 \mathbf{c}, \mathbf{d}}$ indicates the existence of independent finite chains of 10 to 20 magnetic ions. A one-dimensional model is similarly valid for magnetically active polymeric molecular chains of finite or indefinitely great length. ${ }^{5}$

Theoretically the linear chain Heisenberg-Ising model is interesting as one of the simplest many-body systems in which quantum effects play a vital part. This is especially so for the antiferromagnetic chain where the calculation of even the ground-state energy is not easy, although it has been performed exactly. ${ }^{6-9}$ Accurate

3 (a) T. Haseda and A. R. Miedema, Physica 27, 1102 (1961); (b) T. Watanabe and T. Haseda, J. Chem. Phys. 28, 323 (1958); (c) T. Haseda, A. R. Miedema, H. Kobayashi, and E. Kanda, J. Phys. Soc. Japan 17, Suppl. B-I, 518 (1962); (d) H. Kobayashi, T. Haseda, E. Kanda, and S. Kanda, J. Phys. Soc. Japan 18, 349 (1963); (e) L. Berger, S. A. Friedberg, and J. T. Schriempf, Phys. Rev. 132, 1057 (1963).

${ }^{4}$ R. B. Griffiths, Phys. Rev. following paper 135, A659 (1964).

${ }^{5}$ V. L. Ginsburg and V. M. Fain, Dokl. Acad. Nauk SSSR 131, 785 (1960); L. A. Blyumenfel'd, A. E. Kalmanson, and S. P'ei-Ken, ibid. 124, 1144 (1959); L. A. Blyumenfel'd and V. A. Benderskii, ibid. 133, 1451 (1960) [English transls. : Soviet Phys.-Doklady 5, 328 (1960); 4, 260 (1959); 5, 919 (1961)]; L. A. Blyumenfel'd, Biofizika 4, 515 (1959) [English transl.: Biophysics USSR 4, 3 (1959)]

${ }^{6}$ H. A. Bethe, Z. Physik 71, 205 (1931)

${ }^{7}$ L. Hulthén, Arkiv Mat. Astron. Fysik 26A, No. 11 (1938).

${ }^{8}$ R. L. Orbach, Phys. Rev. 112, 309 (1958).

${ }^{9}$ L. R. Walker, Phys. Rev. 116, 1089 (1959). 
values for other properties would be valuable as an aid to judging various approximate theoretical treatments which are also applicable to more complex systems. From the viewpoint of statistical mechanics, it is also of interest to study how the limiting behavior for infinite systems is approached by finite systems. Such insight is useful since, with the advent of fast digital computers, it is feasible to perform exact or Monte Carlo calculations on finite models of many types of physical system.

With this motivation we have undertaken a numerical study of finite chains and rings of $N$ spins with the Hamiltonian,

$$
\begin{array}{r}
\mathfrak{H C}=-2 J \sum_{i=1}^{N}\left\{S_{i}^{z} S_{i+1}{ }^{z}+\gamma\left(S_{i}^{x} S_{i+1}^{x}+S_{i}{ }^{y} S_{i+1} y\right)\right\} \\
-g \beta \sum_{i=1}^{N} \mathbf{H} \cdot \mathbf{S}_{i}
\end{array}
$$

for $\operatorname{spin} S=\frac{1}{2}$. We have computed the energy levels and eigenvectors and thence the thermal and magnetic properties for varying anisotropy $\gamma$ and varying magnetic field $H$ for rings of size $N=2-11$ (and for some open chains) for both ferro- and antiferromagnetic coupling $(J=+|J|$ and $J=-|J|$, respectively). (Our results for the antiferromagnetic entropy in a magnetic field for varying $\gamma$ have already been reported and discussed in the light of the third law of thermodynamics. ${ }^{10}$ )

Previously, Orbach ${ }^{11}$ had computed the energy levels and eigenvectors for $N=2,4,6,8$ and (unpublished) $N=10$ for the case $\gamma=1$, and for some intermediate $\gamma$ values in the case of $N=8$ (also unpublished), but he did not compute the thermal or magnetic properties. Independently of the present authors Griffiths ${ }^{12}$ has calculated the energy levels for $N=3,5,7,9$, and 10 for $\gamma=1$. He evaluated the energies, entropies, specific heats, and susceptibilities in zero field for $N=2-10$, and compared them with the series expansions. We are deeply indebted to both Dr. R. L. Orbach and Dr. R. B. Griffiths for sending us their own unpublished calculations and for helpful discussions and correspondence.

Theoretical work on the Hamiltonian (1.1) with $\gamma=1$ dates back to 1930 when Bloch $^{13}$ introduced the concept of a spin wave and gave the exact eigenstates for one overturned spin $\left(\sum S_{i}{ }^{z}=N S-1\right)$ for ferromagnetic interactions. Using a similar approach, Bethe ${ }^{6}$ obtained the exact eigenstates corresponding to interacting spin waves for an arbitrary number of overturned spins on a linear chain $(\gamma=1)$. He showed how the states may be classified as "unbound" or "bound." Hulthén, in a comprehensive paper, ${ }^{7}$ used Bethe's solutions to

${ }^{10}$ J. C. Bonner and M. E. Fisher, Proc. Phys. Soc. (London) 80, 508 (1962).

${ }^{11}$ R. L. Orbach, Ph.D. dissertation, University of California, 1959 (unpublished); Phys. Rev. 115, 1181 (1959).

${ }_{12}$ R. B. Griffiths, mimeographed reports, Stanford University, 1961, and La Jolla, California, 1963 (unpublished).

${ }^{13}$ F. Bloch, Z. Physik 61, 206 (1930). obtain an integral equation from which he obtained the exact value for the antiferromagnetic ground-state energy of the infinite linear chain. He also obtained the exact ground states for rings of $N=4,6,8$, and 10 spins and estimated the susceptibility at $T=0$.

The arguments of Bethe and Hulthén were extended by $\mathrm{Orbach}^{8}$ to the full anisotropic Hamiltonian (1.1), which had also been considered by Kasteleyn. ${ }^{14}$ Kasteleyn used a variational method which indicated a critical anisotropy constant $\gamma_{c}=0.483$ beyond which the zero-temperature long-range order (present when $\gamma=0$ ) vanished identically. The variational short-range order and energy showed singularities in $\gamma$ at the same point. With the aid of an exact integral equation Orbach showed that these latter singularities were spurious but he was unable to calculate the long-range order exactly. Walker ${ }^{9}$ in an important paper, obtained an analytic solution of Orbach's integral equation which revealed that the antiferromagnetic ground state $E_{0}(\gamma)$ as a function of $\gamma$ was nonanalytic at $\gamma=1$. This implies that the Heisenberg limit $(\gamma=1)$ is a special point perhaps analogous to a critical point. ${ }^{15}$ Walker also gave perturbation series in powers of $\gamma^{2}$ for the energy and for the short-range and long-range order. His series for the long-range order seems to indicate that it does not vanish for $\gamma$ less than about 0.9 .

Perturbation calculations equivalent to those of Walker have been presented by Boon, ${ }^{16}$ who also considered two- and three-dimensional lattices. Alternative perturbation procedures have been proposed by Davis, Rodriguez, Frank, Mannari, and Mills, Kenan, and Korringa ${ }^{17-19}$ Ruijgrok and Rodrigue $z^{20}$ developed a variation method which was rather accurate for the ground state but which yielded a finite long-range order even for $\gamma=1$.

Earlier calculations based on the spin-wave approach have been reviewed by Van Kranendonk and Van Vleck. ${ }^{21}$ One of the most striking predictions is that for a pure Heisenberg antiferromagnet the lowest energy states should obey a dispersion law of the form

${ }^{14}$ P. W. Kasteleyn, Physica 18, 104 (1952).

${ }^{15}$ The fact that $\gamma=1$ is a mathematical singularity (nonanalytic point) of $E_{0}(\gamma)$, follows immediately from Walker's observation that his formula exhibits a pole in any interval, however small, of the open segments $|\gamma|>1$. It should be noted, however, that the singularity at $\gamma=1$ is not visible in graphs of $E_{0}(\gamma)$ for $\gamma \leqslant 1$ contrary to what might be expected. One may regard Kasteleyn's variational transition point $\gamma_{0}=0.48$ (where the energy was nonanalytic as in any phase transition) as an approximation to the "true transition point" at $\gamma=1$.

${ }^{16}$ M. H. Boon, Nuovo Cimento 21, 885 (1961).

${ }^{17}$ H. L. Davis, Phys. Rev. 120, 789 (1960). These results are presented as a power series in $\gamma(=1-\alpha$ in Davis's notation), but the higher coefficients are inexact since the perturbation Hamiltonian is not just the transverse part of (1.1).

${ }^{18}$ S. Rodriguez, Phys. Rev. 116, 1474 (1959); R. L. Mills, R. P. Kenan, and J. Korringa, Physica 26, S204 (1960).

${ }^{19}$ D. Frank, Z. Physik 146, 615 (1956); I. Mannari, Progr. Theoret. Phys. (Kyoto) 19, 201 (1958).

${ }^{20} \mathrm{~T}$. W. Ruijgrok and S. Rodriguez, Phys. Rev. 119, 596 (1960).

${ }_{21}$ J. Van Kranendonk and J. H. Van Vleck, Rev. Mod. Phys. 30, 1 (1958). 
$\epsilon(k)=E-E_{0} \sim|\sin k| .^{21-24}$ This conclusion was tested against the numerical spectra for finite rings of $N=6$ and 8 spins by Mattheiss, ${ }^{25}$ who found an approximately $|\sin k|$ dependence, but with a modified amplitude. More recently, des Cloizeaux and Pearson ${ }^{26}$ extended the computation to the lowest antiferromagnetic states for rings of 16 and 48 spins. They were also able to derive the limiting dispersion law $\epsilon(k)=\pi|J||\sin k|$ for the corresponding class of states of an infinite chain.

The theoretical knowledge of the ground and lower excited states of Heisenberg-Ising chains is not matched by a corresponding knowledge of the thermodynamic behavior. For example, the positions of the maxima in the specific heat and susceptibility do not previously seem to have been estimated with any accuracy. (Although recently, Bulaevskiii ${ }^{27}$ has reported a finite temperature Hartree-Fock calculation which reproduces the general features quite well.)

It is interesting, however, that the spin- $\frac{1}{2}$ model obtained by deleting the parallel or Ising terms $S_{i}{ }^{z} S_{i+1}{ }^{z}$ from Eq. (1.1) to leave only the transverse Hamiltonian is completely soluble. Lieb, Schultz, and Mattis ${ }^{28}$ investiagated the spectrum in detail while Katsura ${ }^{29}$ studied the thermal and magnetic properties including the behavior in a field. Although the transverse Hamiltonian is somewhat artificial, the behavior of this model resembles in many respects the results we have found with the full Hamiltonian (1.1).$^{30}$

It is also possible to calculate by elementary methods the zero-field free energy, correlations and susceptibility for isotropic Heisenberg chains in the limit of infinite spin. ${ }^{31}$ The low-temperature behavior of the thermal properties in this case is somewhat unrealistic since in the classical limit $S=\infty$ the specific heat necessarily goes to a nonzero value as $T \rightarrow 0$. The susceptibility, however, correlates quite closely with the results we find for the $S=\frac{1}{2}$ Heisenberg chains (except that a nonphysical contradiction of the third law is shown again by a nonzero slope for small $T$ ).

The plan of this paper is as follows: In Sec. 2, the machine computations are outlined and the possibilities of extrapolation from finite $N$ to the limit $N \rightarrow \infty$ are illustrated by examining the antiferromagnetic ground state as a function of $\gamma$. The thermal properties, in particular the specific heat, are discussed in Sec. 3 for

\footnotetext{
${ }^{22}$ P. W. Anderson, Phys. Rev. 86, 694 (1952).

${ }^{23}$ R. Kubo, Phys. Rev. 87, 568 (1952).

${ }^{24}$ T. Oguchi, Phys. Rev. 117, 117 (1960).

${ }^{25}$ L. F. Mattheiss, Phys. Rev. 123, 1209 (1961).

${ }^{26} \mathrm{~J}$. des Cloizeaux and J. J. Pearson, Phys. Rev. 128, 2131 (1962).

${ }_{27}^{27}$ L. N. Bulaevskii, Zh. Eksperim. i Teor. Fiz. 43, 968 (1962); see also V. L. Ginsburg and V. M. Fain, ibid. 39, 1323 (1960); 42, 180 (1962) [English transls.: Soviet Phys.-JETP 16, 685 (1963); 12, 923 (1961); 15, 131 (1962)].

${ }_{28}$ E. Lieb, T. Schultz and D. Mattis, Ann. Phys. (N. Y.) 16, 407 (1961).

${ }^{29}$ S. Katsura, Phys. Rev. 127, 1508 (1962).

${ }^{30} \mathrm{~S}$. Katsura (preprint) has discussed the full Hamiltonian by treating the parallel terms as a perturbation.

${ }^{31}$ M. E. Fisher, Am. J. Phys. 32, 343 (1964).
}

antiferromagnetic and ferromagnetic rings. The magnetic properties are described in Sec. 4. Attention is given especially to the magnetization curve at $T=0$ and the corresponding parallel and perpendicular susceptibilities. In Sec. 5 details of the energy spectrum are discussed and the antiferromagnetic anisotropy gap and the asymptotic degeneracy of the ground state are estimated. The spin-wave dispersion laws for ferro- and antiferromagnetic chains are illustrated. The shortrange antiferromagnetic order and further pair correlation functions $\left\langle S_{0}{ }^{z} S_{l}{ }^{z}\right\rangle$ are described in Sec. 6. The long-range order is estimated by extrapolation and compared with previous approximations. Our results are for the most part presented graphically but tables of the thermal and magnetic properties for $\gamma=1$ and 0.5 and $N=9,10$, and 11 have been prepared and will be made available on request to the authors. (For reasons of economy they are not reproduced in this article.)

\section{FINITE CHAIN CALCULATIONS AND THE ANTI- FERROMAGNETIC GROUND STATE}

For a finite system of $N\left(S=\frac{1}{2}\right)$ spins the problem of calculating the energy levels reduces to the diagonalization of the $2^{N} \times 2^{N}$ matrix representing the Hamiltonian. (As basic states it is covenient to use direct products of "UP" and "DOwN" single-spin states: These are, of course, eigenstates of the Ising Hamiltonian $\gamma=0$.) By classifying the states by the value of $S^{z}$, the $z$ component of the total spin, the matrix splits into $N+1$ blocks of order $1, N,\left(\begin{array}{c}N \\ 2\end{array}\right), \cdots\left(\begin{array}{c}N \\ \frac{1}{2} N\end{array}\right), \cdots N, 1$ ( $N$ even). A further reduction can be obtained for closed chains (rings) of spins by using the translational invariance. Each level is then also classified by a (total) wave number $k=2 \pi r / N$ with $r=0,1,2, \cdots, N-1$ ( $k$ is only determined up to a multiple of $2 \pi$ ).

The largest value of $N$ which can be handled numerically is limited by the size of the largest block matrix in relation to the speed and capacity of the electronic computer available. Our calculations were performed on the now relatively slow University of London Ferranti "Mercury" which restricted us to $N=11$ or less. To economize on computing time many of the calculations for intermediate values of $\gamma$ and magnetic field, etc., were, in fact, performed with $N=8$, or 10 , once the trend with increasing $N$ was clear. ${ }^{10}$ The diagonalization, yielding both energy levels and eigenstates, was performed by standard subroutines and the results checked against the other available computations. ${ }^{11,12,32}$ The calculation of the thermodynamic properties was performed by direct evaluation of the appropriately weighted partition sums.

The possibilities of estimating the properties of

${ }^{32}$ For the Heisenberg limit $\gamma=1$ one has a further check since the total spin $S$ is also conserved so that many levels from different blocks coincide. It was not found possible to use this added invariance to reduce the calculations. 
TABLE I. Energy of the antiferromagnetic ground state $\varepsilon_{0}(N)$.

\begin{tabular}{cccc}
\hline \hline$N$ & Even & $N$ & Odd \\
\hline 4 & -1.00000 & 3 & -0.50000 \\
6 & -0.93425 & 5 & -0.74721 \\
8 & -0.91277 & 7 & -0.81577 \\
10 & -0.90309 & 9 & -0.84384 \\
12 & -0.8979 & 11 & -0.85799 \\
$\vdots$ & -0.88629 & $\infty$ & -0.88629 \\
\hline
\end{tabular}

infinite chains from those of finite chains are revealed by a study of the pure Heisenberg antiferromagnetic ground state energies. The antiferromagnetic ground state $E_{0}$ is equal to $-2 N|J| \ln 2+\frac{1}{2} N|J|$ for the Hamiltonian (1.1) with $H=0$ and $\gamma=1$ in the limit $N \rightarrow \infty$. It is convenient to define normalized groundstate energies for finite $N$

$$
\mathcal{E}_{0}(N)=E_{0}(N) / N|J|
$$

and these energies are tabulated numerically in Table I. (The values for $N=4,6,8$, and 10 were given exactly by Hulthén, ${ }^{7}$ that for $N=12$ by Ledinegg and Urban. ${ }^{33}$ ) In Fig. 1 the energies are plotted versus $1 / N$ (circles) and versus $(1 / N)^{2}$ (squares). The values form two sequences ( $N$ odd or even) and it is evident that the limiting energy is approached linearly with $(1 / N)^{2}$. A relation of the form

$$
\mathcal{E}_{0}(N)=\mathcal{E}_{0}(\infty)+a / N^{2}
$$

holds quite accurately even down to $N=4$ or 5 with $a_{\text {odd }} \simeq-2 a_{\text {even }}$.

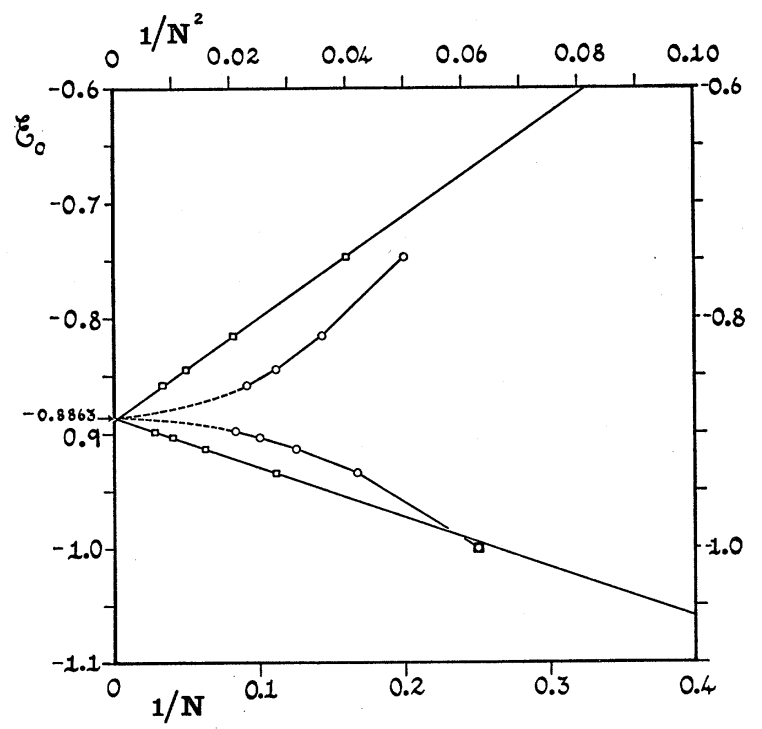

FIG. 1. Antiferromagnetic ground-state energies versus $1 / N$ (circles) and versus $(1 / N)^{2}$ (squares) for pure Heisenberg rings $(\gamma=1)$.

${ }^{33}$ E. Ledinegg and P. Urban, Acta"Phys. Austriaca 6, 227 (1953).

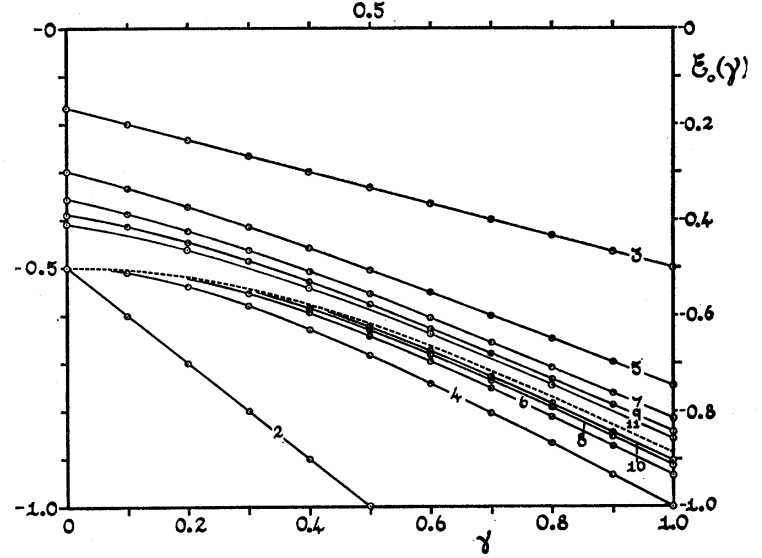

Fig. 2. Antiferromagnetic ground state as a function of $\gamma$ for finite and infinite rings. The exact limiting curve due to Orbach (Ref. 8 ) is shown by the dashed curve.

If the limiting value had not been known, it could have been estimated to about $0.1 \%$ accuracy by linear extrapolation with $1 / N^{2}$. Heisenberg chains with open ends show a slower convergence, apparently linear with $1 / N$. For pure Ising $(\gamma=0)$ rings the ground state is exact for $N$ even but approaches the limit as $1 / N$ for $N$ odd on account of the "misfit seam." In Fig. 2 the approach of the antiferromagnetic ground state to the limit is shown as a function of $\gamma$ for $N=2-11$. The limiting curve $\left(\right.$ due to $\mathrm{Orbach}^{8}$ ) is rather well defined by these results.

Encouraged by the relatively simple and regular behavior of the finite $N$ results found here, we may go on to examine properties for which the exact $N=\infty$ limits are unknown with the reasonable expectation that careful extrapolation to large $N$ will not be misleading.

\section{THERMAL PROPERTIES}

In this section we consider the thermal properties firstly of antiferromagnetic and then of ferromagnetic chains.

\section{Antiferromagnetic Coupling}

In Fig. 3 is shown the energy per spin in zero field as a function of temperature for antiferromagnetic pure Ising and Heisenberg rings of $N=2-11$ spins. As for the ground states, odd and even rings form two distinct sequences. For the Ising case $(\gamma=0)$, where the exact limit is known, the two sequences approach the limiting curve monotonically from above and below. There seems no reason to doubt that the same situation prevails at $\gamma=1$. The energy of an infinite Heisenberg chain is thus defined to an accuracy of better than $\pm 0.5 \%$ down to temperatures of $k T /|J|=0.5$ by the mean of the curves for $N=10$ and 11 . (These two curves and the estimated limiting curve have been tabulated and are available on request.) Below this temperature the true limiting curve is less certain, but since the value for 


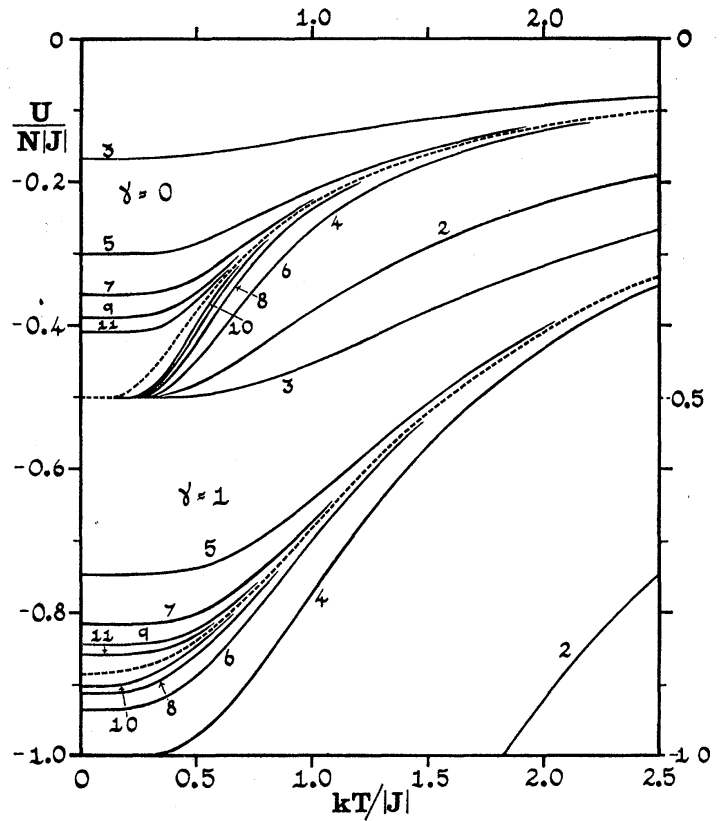

Fig. 3. Zero-field energy as a function of temperature for finite antiferromagnetic Ising and Heisenberg chains. The limiting $(N=\infty)$ curve is shown dashed in each case.

$T=0$ is known exactly, even a roughly estimated limiting curve would be accurate to $\pm 1 \%$. We return to this point shortly in considering the entropy.

The entropy for the pure antiferromagnetic Heisenberg chains is shown in Fig. 4 . The convergence appears

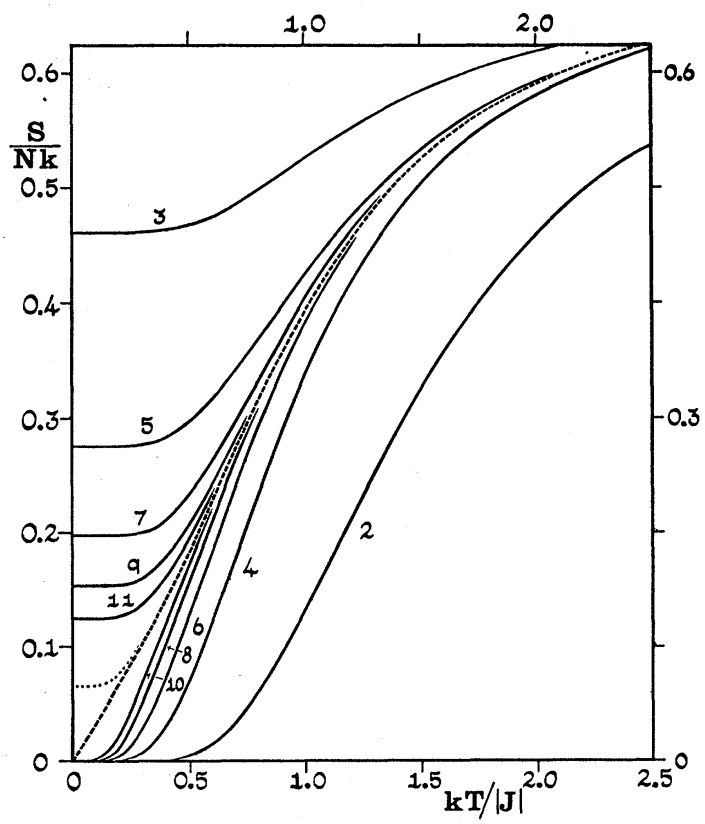

Fig. 4. Entropy versus temperature for antiferromagnetic Heisenberg chains $(\gamma=1)$. The dotted curve is the mean of $N=10$ and $N=11$ weighted as in (a) (Ref. 35), and the dashed curve is the estimated limit. to be similar to that for the energy and the limit seems well determined down to $k T /|J|=0.5$ by interpolation between the curves for odd and even $N$. For odd $N$ at $T=0$ the ground state is fourfold degenerate and the entropy per spin thus goes to zero only as $2 k \ln 2 / N$.

To estimate the nature of the low-temperature behavior for $N \rightarrow \infty$, we may reasonably postulate a power law for the limiting free energy per spin (or, more directly, for the limiting internal energy). Thus, suppose $^{34}$

$$
U(T)-U(0)=A N|J|(k T /|J|)^{\alpha},
$$

where $A$ and $\alpha$ are fixed (as $T \rightarrow 0$ ). Then for the specific heat we have

$$
C / N k=A \alpha(k T /|J|)^{\alpha-1}
$$

and for the entropy

$$
S(T) / N k=A \alpha(\alpha-1)^{-1}(k T /|J|)^{\alpha-1} .
$$

By combining (3.1) and (3.3) we obtain

$$
U(T)-U(0)=\left(1-\alpha^{-1}\right) T S(T)
$$

so that a plot of energy $U / N|J|$ versus $T S / N|J|$ for $N \rightarrow \infty$ should be linear with slope determined by the index $\alpha$. Such a plot is shown in Fig. 5 for $N=10$ and $N=11$ and supports the view that the limiting curve would indeed be linear. The solid line which is drawn from the exact $(N \rightarrow \infty)$ ground state with slope 0.515 should be close to any reasonable "best" fit and yields the estimate $\alpha \simeq 2.06 \pm 0.03$. This value is largely determined by the data for temperatures in the range $k T /|J|=0.30-0.60$. The dashed line, on the other hand, is of slope $\frac{1}{2}$ and corresponds to $\alpha=2$ exactly. It seems likely that this is the true limiting value which would be obtained by fitting data for larger $N$ at lower temperatures. ${ }^{35}$

If we assume $\alpha=2$, the amplitude $A$ may be estimated by various methods; for example, from the temperature variation of $U / N|J|$ and $T S / N|J|$, from plots of $U / N|J|$ versus $(S / N k)^{2}$ using the weighted means, ${ }^{35}$ and from the slope of the weighted entropy means at low temperatures. We thus obtain the approximate results

$$
\begin{aligned}
& U(T) \approx U(0)+0.175 N|J|(k T /|J|)^{2}, \\
& S(T) \approx 0.35 N k(k T /|J|), \quad(\gamma=1)
\end{aligned}
$$

valid up to $k T /|J|=0.4$ to $0.5 .^{36}$ These are shown as

${ }^{34}$ Our method is a slight modification of a procedure devised by Griffiths (Ref. 12) who plotted $U(T)$ versus $S(T)$ and estimated the index $\alpha$ by fitting $B$ and $\beta$ in the power law $U-U(0)=B S^{\beta}$.

${ }^{35}$ Griffiths (Ref. 12) estimated $\alpha=2.1$, but also suggested that $\alpha=2$ was probably the exact value. This conclusion is supported more closely by analyzing the trends of the weighted means (a) $\left[N P_{N}+(N-1) P_{N-1}\right] /(2 N-1)$, (b) $\frac{1}{2} P_{N}+\frac{1}{2} P_{N-1}$, (c) $\left[(N-1) P_{N}\right.$ $\left.+N P_{N-1}\right] /(2 N-1)$ (where $P$ is the thermodynamic property in question), which converge more rapidly.

${ }^{36}$ Our conclusions regarding the amplitude $A$ agree closely with those of Griffiths. The spin-wave theory of the low-temperature behavior is given by R. Kubo, Phys. Rev. 87, 568 (1952); J. Van Kranendonk and J. H. Van Vleck, Rev. Mod. Phys. 30, 1 (1958). 
dashed curves in Figs. 3 and 4 and seem to be the most reasonable estimates of the low-temperature behavior.

It is interesting that the functional forms of (3.5) and (3.6) agree with the predictions of simple antiferromagnetic spin-wave theory but the corresponding amplitude ${ }^{36} A_{\text {s.w. }}=\pi / 6=0.52$ is too great by a factor of about three. A $T^{2}$ law for the energy is exact for the pure transverse Hamiltonian discussed by Katsura. ${ }^{29}$

The corresponding specific heats for pure antiferromagnetic Heisenberg chains are shown in Fig. 6. For temperatures above $k T /|J|=0.5$ the convergence again appears to be monotonic from above and below and the limiting curve (shown dashed) can be estimated quite accurately through the maximum of height

at

$$
C_{\max } / N k \simeq 0.350 \quad(\gamma=1)
$$

$$
k T_{\max } /|J| \simeq 0.962 \quad(\gamma=1) .
$$

At lower temperatures the convergence must be more complex since, for finite $N$, the specific heat always vanishes exponentially fast as $T \rightarrow 0$, owing to the finite energy gap between the ground and first excited states. As $N \rightarrow \infty$, however, the states close up (as $1 / N$ ) and merge into a continuum, which runs from the ground state (see below). The probable limiting low-temperature behavior follows from (3.5) and (3.6), which yield

$$
C(T) \simeq 0.35 N k(k T /|J|) \quad(\gamma=1),
$$

and this has been used in deriving the estimated curve for the limit $N=\infty$ shown by the dashed line in Fig. 6 .

The variation of the antiferromagnetic specific heat with anisotropy may be seen from Fig. 7 which shows the specific heats for rings of $N=8$ spins. The exact limiting curve for $\gamma=0$ and the estimated limit for $\gamma=1$ are plotted as dashed lines to indicate the degree to which the results for $N=8$ approach the limit. The effect of increasing $\gamma$ from the Ising value $\gamma=0$ is to shift the specific heat maximum to higher temperatures, to reduce its height and to broaden the peak. At low temperatures $(k T /|J| \simeq 0.1)$ the curves for finite $N$ (even) and $\gamma$ in the range $0.1-0.6$ display anomalous small peaks and points of inflection. These are "small number effects" due to the finite splitting of the degenerate Ising ground state by the transverse terms in the Hamiltonian. For fixed $\gamma$ and increasing $N$, this splitting diminishes and eventually goes to zero as $N \rightarrow \infty$ (see Sec. 5). Correspondingly, the anomalies move to lower temperatures and are reduced in magnitude, finally disappearing in the limit $N=\infty$.

For $\gamma \neq 1$ and large but finite $N$, the specific heat curves at low temperature (but above the anomalies) vanish exponentially fast, roughly as $\exp \left[-\Delta E_{A}(\gamma) /\right.$ $k T]$, where $\Delta E_{A}(\gamma)$ is the limiting anisotropy gap between the ground state and the first excited states. The value of this gap is estimated in Sec. 5 ; it approaches zero as $\gamma \rightarrow 1$ and the Ising value, $2|J|$, as $\gamma \rightarrow 0$.

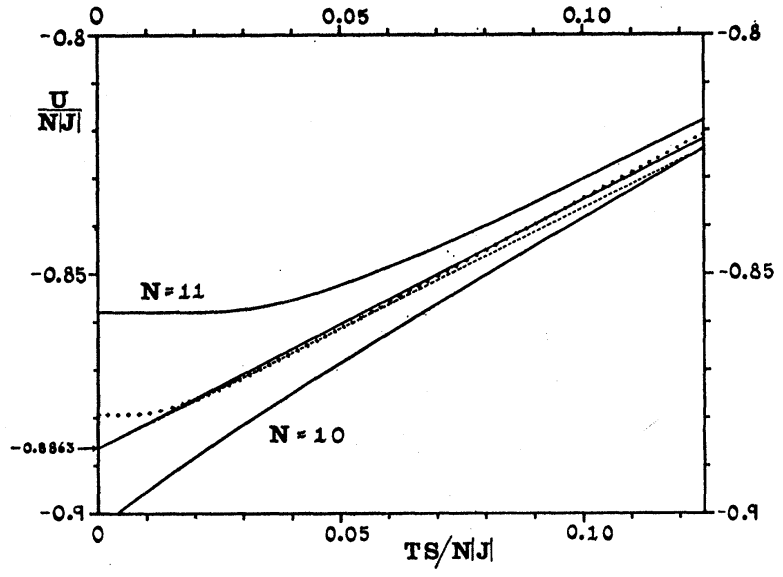

FIG. 5. Energy versus the product of temperature and entropy for $N=10$ and $N=11$ Heisenberg antiferromagnetic chains. The line of crosses is the 11/10 mean weighted as in (a) (Ref. 35).

[Owing to the existence of "bound states" of indefinitely large groupings of adjacent "overturned spins," the number of states just above the gap might be of order $N^{2}$ rather than $N$. Consequently, it is possible that the true limiting specific heats might rise faster than $\exp \left[-\Delta E_{A} / k T\right]$. If this were the case, $\Delta E_{A}(\gamma)$ should be replaced by an "effective gap" $\Delta E_{A} *(\gamma)<\Delta E_{A}(\gamma)$ as happens at the Ising limit $\gamma=0$ where, in fact, one finds that $\Delta E_{A} *(0)=\frac{1}{2} \Delta E_{A}(0)$.]

The variation of specific heat with magnetic field may be studied just as for zero field. The convergence at temperatures above $k T /|J| \simeq 0.3$ is found to be

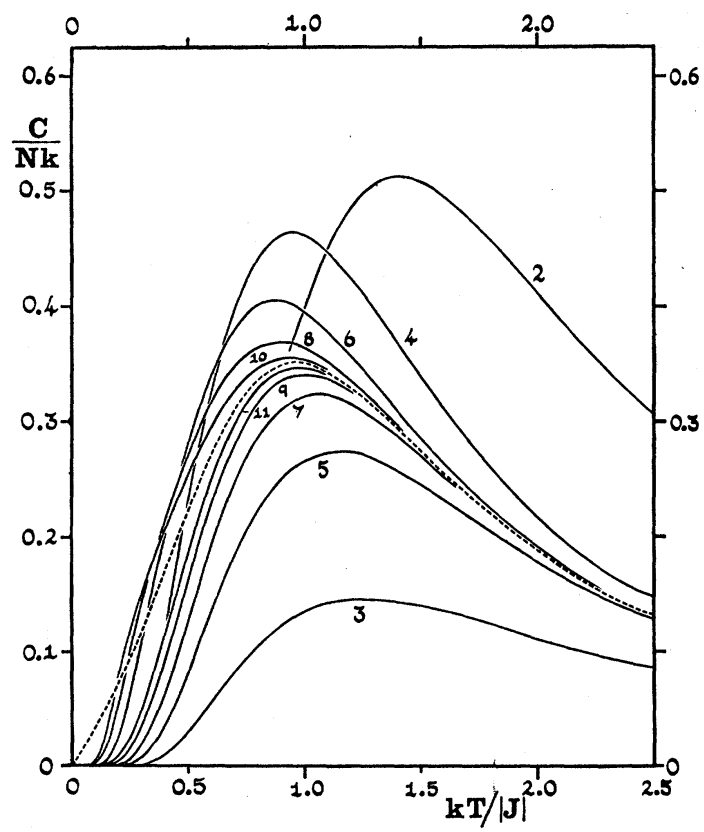

FIG. 6. Variation of specific heat with temperature for antiferromagnetic Heisenberg chains : finite $N$, solid lines; estimated limit $N=\infty$, dashed line. 


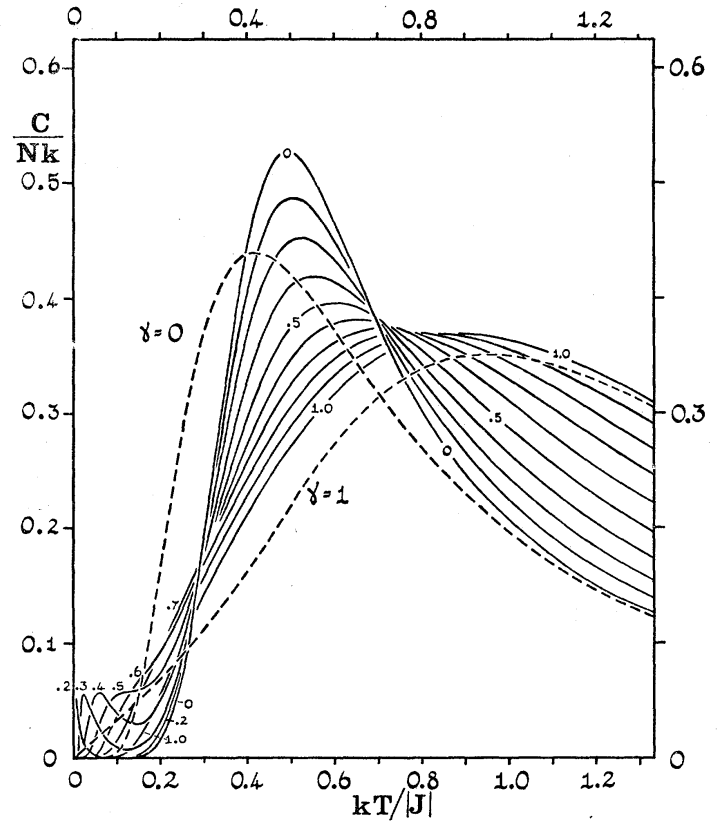

FIG. 7. Antiferromagnetic specific heat for rings of $N=8$ spins for various anisotropies $\gamma$. The dashed curves are the exact and estimated limiting curves $(N=\infty)$ for $\gamma=0$ and $\gamma=1$, respectively.

quite rapid especially if the consecutive means for $N=2 n$ and $N=2 n-1$ are considered. Accordingly, in Fig. 8, we have plotted for the pure Heisenberg case only the mean specific heats for $N=10$ and 9 at various fields (except at $H=0$ where the estimated limit is shown). These means should represent the true limiting curves to within 1 or $2 \%$ down to temperatures of $k T /|J| \simeq 0.5$. With increasing field the maximum shifts to lower temperatures and falls in height. However, at the field $H_{s}(g \beta /|J|)=4$, which represents a "critical field" above which the antiferromagnet becomes fully magnetized at zero temperature (i.e., saturation oc-

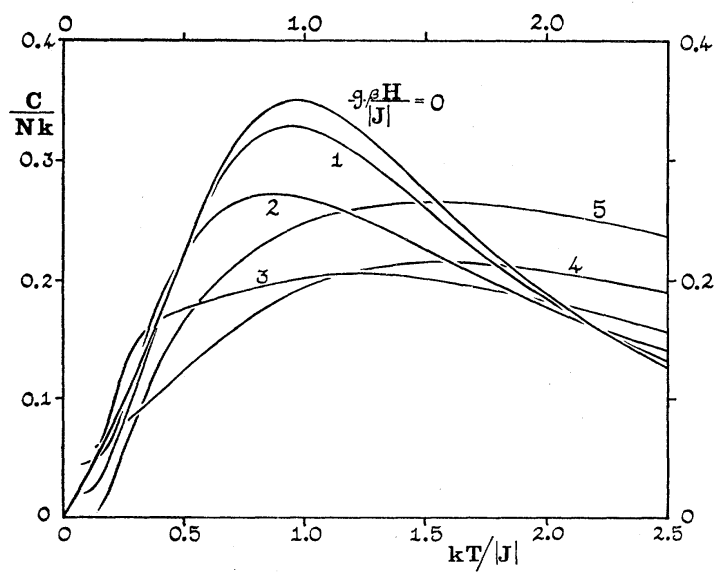

FIG. 8. Variation of the antiferromagnetic specific heat with magnetic field for $\gamma=1$. Except for $H=0$, the curves are the means of the values for $N=9$ and 10 . curs, see Sec. 4), there remains only a broad low maximum at a higher temperature. The behavior is similar for other values of $\gamma$ as can be seen from Fig. 9, where the specific heat for $\gamma=0.5$ is plotted. The critical field in this case is $H_{s}=3(|J| / g \beta)$. Above the critical field the initial rise is governed by the energy gap $\Delta E_{H}(\gamma)=H g \beta-2(1+\gamma)|J|$ and it may be observed that the maximum starts to increase in height again.

\section{Ferromagnetic Coupling}

We turn now to ferromagnetic chains: Values of the energy, entropy, and specific heat for the longest chains and $\gamma=1$ and 0.5 have been tabulated and are available on request. The energy (per spin) is plotted versus the temperature for Heisenberg chains of $N=3,4, \cdots, 10$, 11 spins in Fig. 10. In contrast to the antiferromagnetic case, the curves for finite $N$ do not appear to bracket the limiting curve, but rather increase monotonically

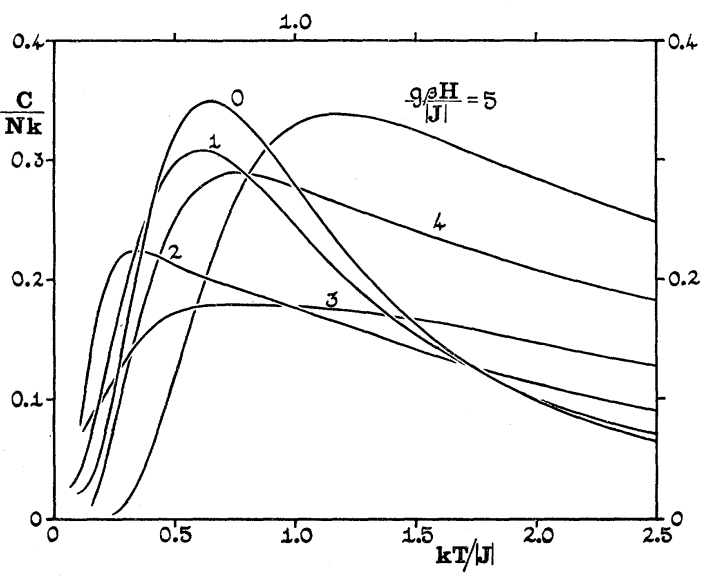

Fig. 9. Variation of the antiferromagnetic specific heat with field as in Fig. 8, but for $\gamma=0.5$.

towards it. This makes extrapolation at low temperatures more difficult although the limit seems quite accurately defined down to $k T / J=0.5$. (All curves, of course, approach the exact limiting ground state $\left.\mathcal{E}=-\frac{1}{2}\right)$.

The curves for the entropy display a more complicated convergence, successive curves crossing at low temperatures. The ground state of the ferromagnet when $\gamma=1$ has spin $N S=\frac{1}{2} N$, and hence degeneracy $N+1$. Consequently, the zero-point entropy is $(k / N) \ln (N+1)$, which approaches zero rather slowly. We may, nonetheless, attempt to estimate the low-temperature behavior of the energy and entropy by the power law analysis presented in Eq. (3.1) to (3.4). The corresponding $U(T)$ versus $T S(T)$ plots are not very straight and their slopes increase monotonically with $N .^{37}$ The maximum slopes for $N=9$ and 10 correspond to an index in (3.1)

37 The curves are concave downwards except for very small values of $T S / N J$ (below 0.04 ), where an exponential decay sets in. 
of $\alpha=1.42$ and 1.43 , respectively, while the slopes of the tangents through the origin correspond to $\alpha=1.312$ and 1.324. Rough extrapolation linearly in $1 / N$ suggests that the true index might lie in the neighborhood of $\alpha=1.45$ 1.50 , but the corresponding pure power laws would only be followed closely below temperatures of $k T / J \simeq 0.25$. Simple spin-wave theory predicts $\alpha=\frac{3}{2}$, and one might expect this to be accurate since the ground and first excited states are described exactly in the ferromagnetic case. However, as is known, spin-wave theory does not give any account of the lower lying "bound" states which arise for further overturned spins. Nevertheless, our results do indicate that $\alpha=\frac{3}{2}$ might be exact. Accepting this, one may estimate the amplitudes

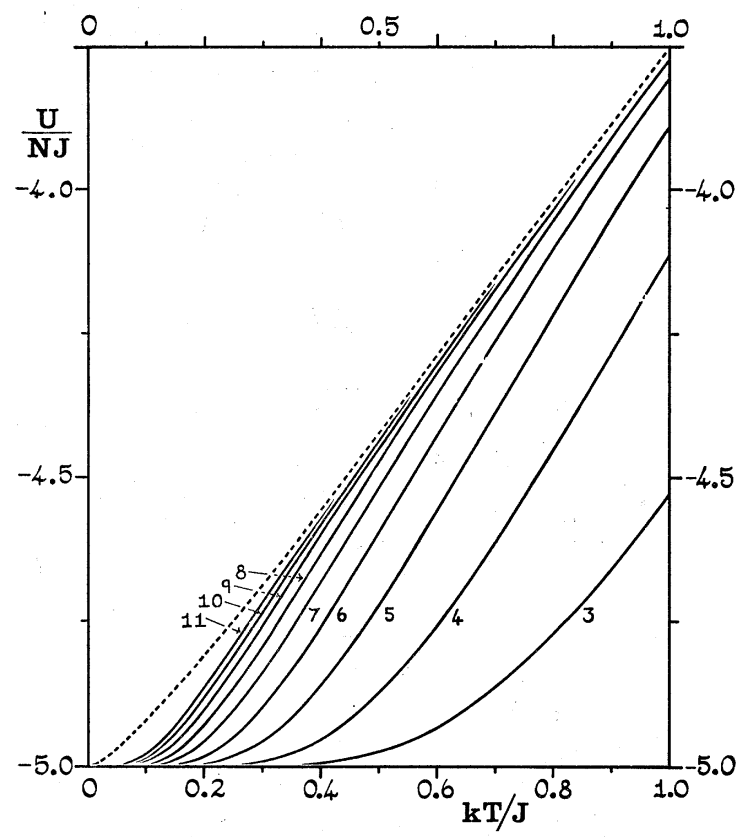

FIG. 10. Energy of ferromagnetic Heisenberg chains $(\gamma=1)$ for increasing $N$.

roughly which yields

$$
\begin{gathered}
U(T) \simeq 0.28 N J(k T / J)^{3 / 2}, \quad(T \rightarrow 0) \\
S(T) \simeq 0.85 N k(k T / J)^{1 / 2} .
\end{gathered}
$$

The amplitudes predicted by spin-wave theory are larger by a factor of about 1.3 .

The specific heats for the Heisenberg rings are shown in Fig. 11 as solid curves. The convergence is monotonic decreasing at temperatures above $k T / J=0.3$ for $N \geqslant 8$.

It seems clear that a double maximum will develop when $N=13$ or 14 and that the maximum of the limiting curve will not derive from the maxima in the curves for $N \leqslant 11$. The broken curves in Fig. 11 correspond to open chains of 3 and 4 spins. Although they do not seem to converge very rapidly, they do approach the limit from below and seem to give a truer representa-

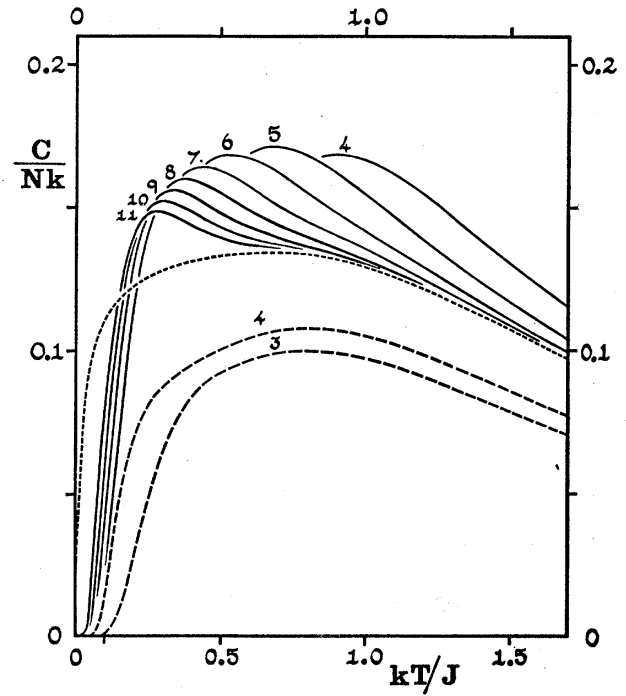

FIg. 11. Specific heats for ferromagnetic Heisenberg rings (solid curves) and open chains (broken curves). The dashed curve is the estimated limiting curve.

tion of its shape. (Calculations for longer open chains were not performed since, as translational symmetry is absent, they would have required more machine time than available.) Guided by these results and the lowtemperature estimates following from (3.10) [which indicate that $\left.C(T) \sim T^{1 / 2}\right]$ and remembering that the integrated area under the specific heat curve must check with the energy one may estimate with moderate accuracy the limiting ferromagnetic specific heat curve.

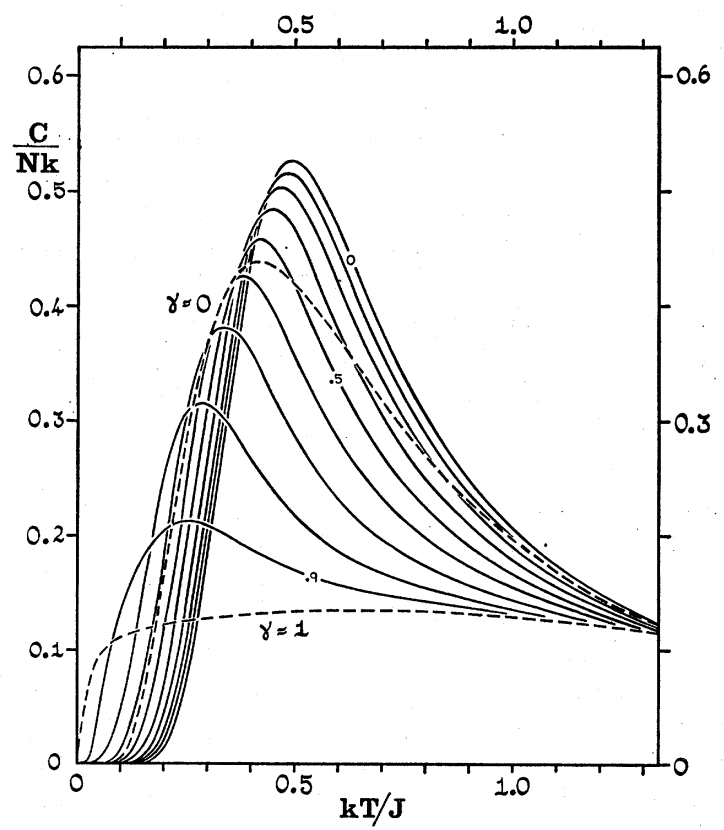

FIG. 12. Variation of ferromagnetic specific heats with anisotropy for rings of $N=8$. (For $\gamma=0$ and 1 , the exact and estimated limiting curves are also plotted.) 


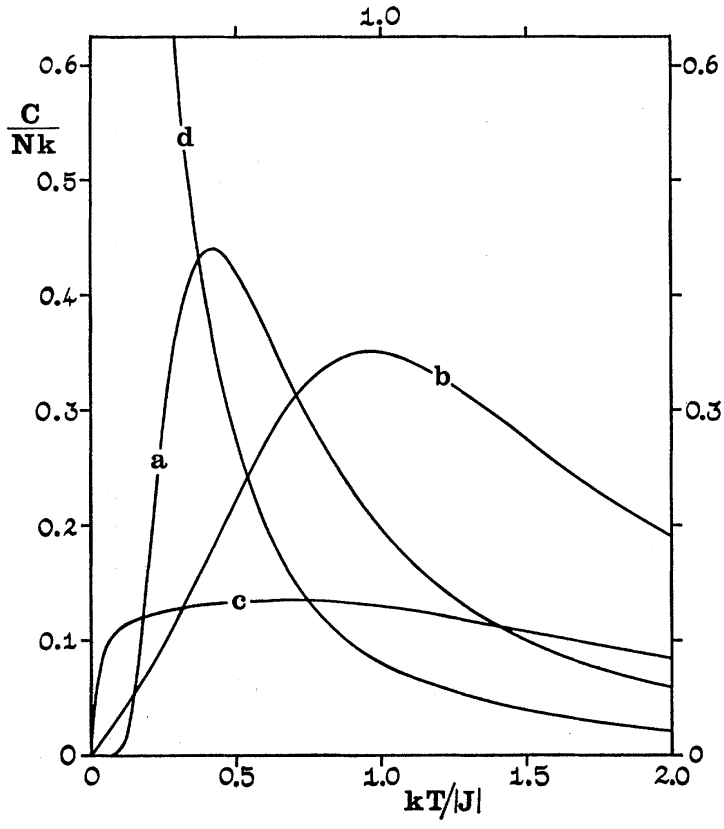

FIG. 13. Comparison of specific heats for infinite chains for (a) $S=\frac{1}{2}$ Ising coupling, (b) $S=\frac{1}{2}$ Heisenberg antiferromagnetic coupling, (c) $S=\frac{1}{2}$ Heisenberg ferromagnetic coupling, and (d) $S=\infty$ Heisenberg coupling (classical spins).

This is shown dashed in Fig. 11; the rather broad peak has a maximum height

at

$$
C_{\max } / N k \approx 0.134 \text {, }
$$

$$
k T_{\max } / J \approx 0.70 .
$$

The nature of the variation of specific heat with anisotropy can be gauged from Fig. 12, which (except for $\gamma=1$ where the estimated limit is plotted) shows the results for rings of $N=8$ spins at intervals of $\Delta \gamma=0.1$. The effects of a magnetic field (for $\gamma=1$ ) are rather similar to those due to anisotropy so that we do not present a figure. With a field $H(g \beta / J)=0.5$, the specific heat maximum increases sharply to a height of about $C / N k=0.37$ at $(k T / J)=0.61$. Further increase of the field to $H(g \beta / J)=1.0$ and 2.0 increases the maximum to $C / N k=0.42$ and 0.45 and raises the corresponding temperatures to $(k T / J)=0.94$ and 1.45 , respectively. These figures are derived for $N=8$ but should not differ significantly from the limiting results since convergence is faster when $H \neq 0$ or $\gamma \neq 1$, since the ferromagnetic ground state is then nondegenerate, and is separated by a finite energy gap, even in the limit $N=\infty$.

Finally, in Fig. 13 are compared on the same scale the exact and estimated specific heats for infinite chains with (a) $S=\frac{1}{2}$ Ising coupling, ferro- or antiferromagnetic, (b) $S=\frac{1}{2}$ Heisenberg antiferromagnetic coupling, (c) $S=\frac{1}{2}$ Heisenberg ferromagnetic coupling, and (d) $S=\infty$ (classical) Heisenberg ferro- or antiferromagnetic coupling. ${ }^{31}$ The large difference between the relatively sharp antiferromagnetic $S=\frac{1}{2}$ Heisenberg specific heat and the low broad ferromagnetic specific heat is striking testimony to the lower stability of the isotropic ferromagnetic coupling. Curve (d) for $S=\infty$ continues to rise monotonically to a maximum at $T=0$ of height $C / N k=1$.

\section{MAGNETIC PROPERTIES}

\section{Antiferromagnetic Coupling}

In Fig. 14 are plotted the antiferromagnetic susceptibilities for isotropic Heisenberg coupling $(\gamma=1)$ in zero field for finite rings of $N=3,4,5, \cdots, 11$ spins. (The tabulated values for $N=10$ and 11 are available.) The limiting curve is apparently bracketed by the curves for odd $N$, which approach monotonically from above, and those for even $N$, which approach monotonically from below. The convergence is rather rapid above $k T /|J|=0.6$, at which temperature the values for $N=10$ and 11 differ by only $5 \%$, and their mean probably differs from the true limiting curve by less than $1 \%$. The susceptibility displays a rounded maximum of height (for $N \rightarrow \infty$ )

at

$$
\chi_{\max } /\left(g^{2} \beta^{2} /|J|\right) \simeq 0.07346, \quad(\gamma=1)
$$

$$
k T_{\max } /|J| \simeq 1.282 . \quad(\gamma=1) .
$$

The situation at low temperatures as zero is approached is more complicated. For finite chains with $N$ even, the antiferromagnetic ground state for all values

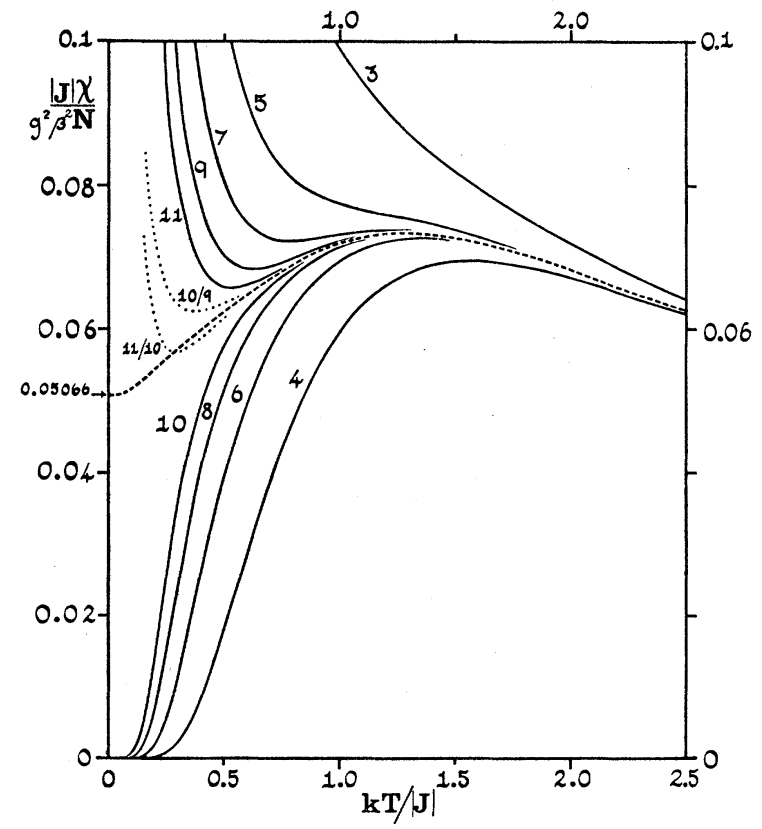

FIG. 14. Antiferromagnetic susceptibility versus temperature for finite Heisenberg rings (solid curves) and the estimated limit for infinite rings (dashed curve). The dotted curves are means of $N=9$ and 10 , and $N=10$ and 11 weighted as in (a) and (c) (Ref. 35), respectively. 
of $\gamma$ is characterized by total spin component $S^{z}=0$, and there is a finite gap to the first excited state of nonzero $S^{z}$. Consequently, for all chains of even $N$ (finite), the susceptibility parallel to the $z$ axis approaches zero exponentially fast as $T \rightarrow 0$, at a rate governed by the energy gap. On the other hand, for finite odd $N$, the ground state, or rather degenerate ground states, have $S^{z}= \pm \frac{1}{2}$. (Clearly, $S^{z}=0$ is impossible.) The susceptibility for odd $N$ thus diverges as $1 / T$ as $T$ approaches zero. However, as $N$ increases the (relative) amplitude of the divergence falls in magnitude, and it sets in at a lower temperature. These remarks may be verified explicitly in the case $\gamma=0$ (Ising limit), where the exact

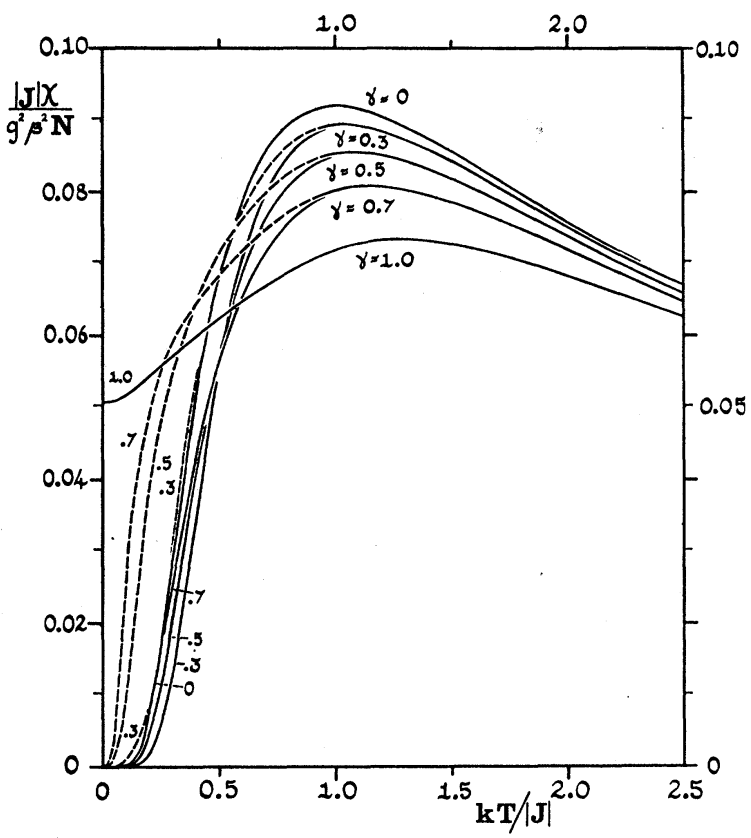

Fig. 15. Variation of the parallel antiferromagnetic susceptibility with anisotropy for rings of $N=8$ spins (solid curves). The estimated limiting values are shown by broken curves. Note that for $\gamma=0$ and $\gamma=1$ only the exact and estimated limiting curves are plotted.

result for the parallel susceptibility of a finite chain is

$$
\chi_{\mathrm{II}}(\gamma=0)=\frac{g^{2} \beta^{2} N}{4 k T} e^{-|J| / k T}\left\{\frac{1-(-\tanh K)^{N}}{1+(-\tanh K)^{N}}\right\}
$$

with

$$
K=|J| / 2 k T \text {. }
$$

In this case, as is well known, the limiting susceptibility, which is approached both for even $N$ and odd $N$ $(T>0)$, goes to zero exponentially fast (see curve for $\gamma=0$ in Fig. 15).

Now for $\gamma$ nonzero, but less than unity, the anisotropy gap between the ground state(s) and first excited states persists even in the limit $N \rightarrow \infty$ (see next section). Consequently, the limiting behavior should be similar to the Ising case with $\chi_{11}(T) \rightarrow 0$ as $T \rightarrow 0$. Figure 15 (a) $\gamma<1$

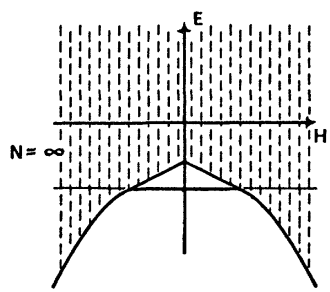

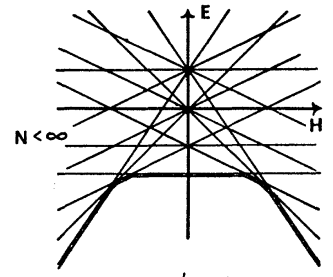

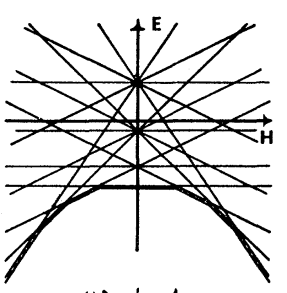

(b) $\gamma \approx 1$

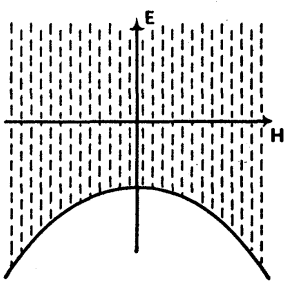

Fig. 16. Sketch of the energy levels versus magnetic field for finite and infinite isotropic $(\gamma=1)$ and anisotropic $(\gamma<1)$ systems (Ref. 39). The shading indicates a continuum of levels.

shows the parallel susceptibilities for rings of $N=8$ spins for $\gamma=0.3,0.5$, and 0.7 (solid lines) and the approximate limiting curves (broken lines) roughly estimated on the basis of the limiting anisotropy gap. These curves should be accurate to within 5 or $10 \%$ down to $k T /|J|$ $=0.3$. Evidently the effect of increasing $\gamma$ is to shift the susceptibility peak to a higher temperature, to reduce its height and to increase its width. The positions of the maxima are for $\gamma=0, \theta_{\max }=k T_{\max } /|J|=1$; for $\gamma=0.3$, $\theta_{\max } \simeq 1.03$, for $\gamma=0.5, \theta_{\max } \simeq 1.07$ and for $\gamma=0.7, \theta_{\max }$ $\simeq 1.14$. (For small $\gamma$ the variation is quadratic in $\gamma$.)

For the isotropic pure Heisenberg case $\gamma=1$, however, the behavior as $T \rightarrow 0$ is different. As $N$ increases, the gap between the ground state and the first excited states with $S^{z} \neq 0$ shrinks and approaches zero as $1 / N .^{38}$ In the limit $N=\infty$, there is no anisotropy gap. This

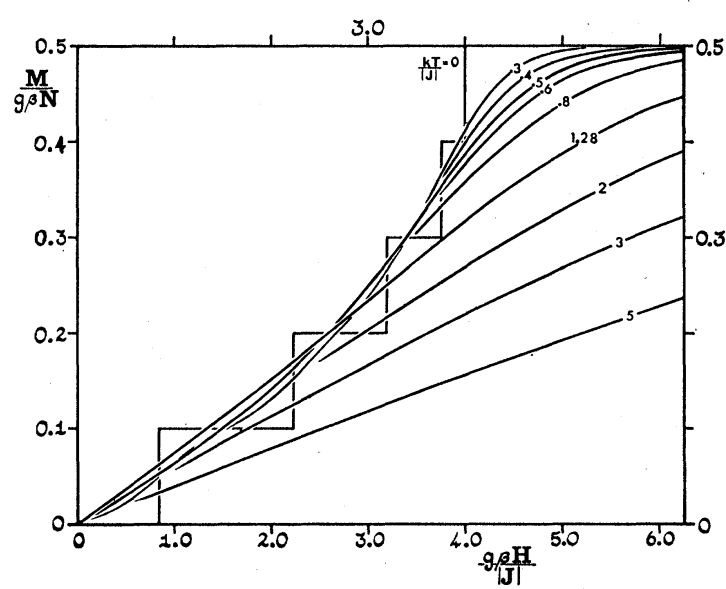

FIG. 17. Magnetization curves for an isotropic antiferromagnetic chain of $N=10$ spins. The numbers on the curves give the appropriate values of $k T /|\boldsymbol{J}|$.

${ }^{38}$ For simplicity we describe only the case for $N$ even. 


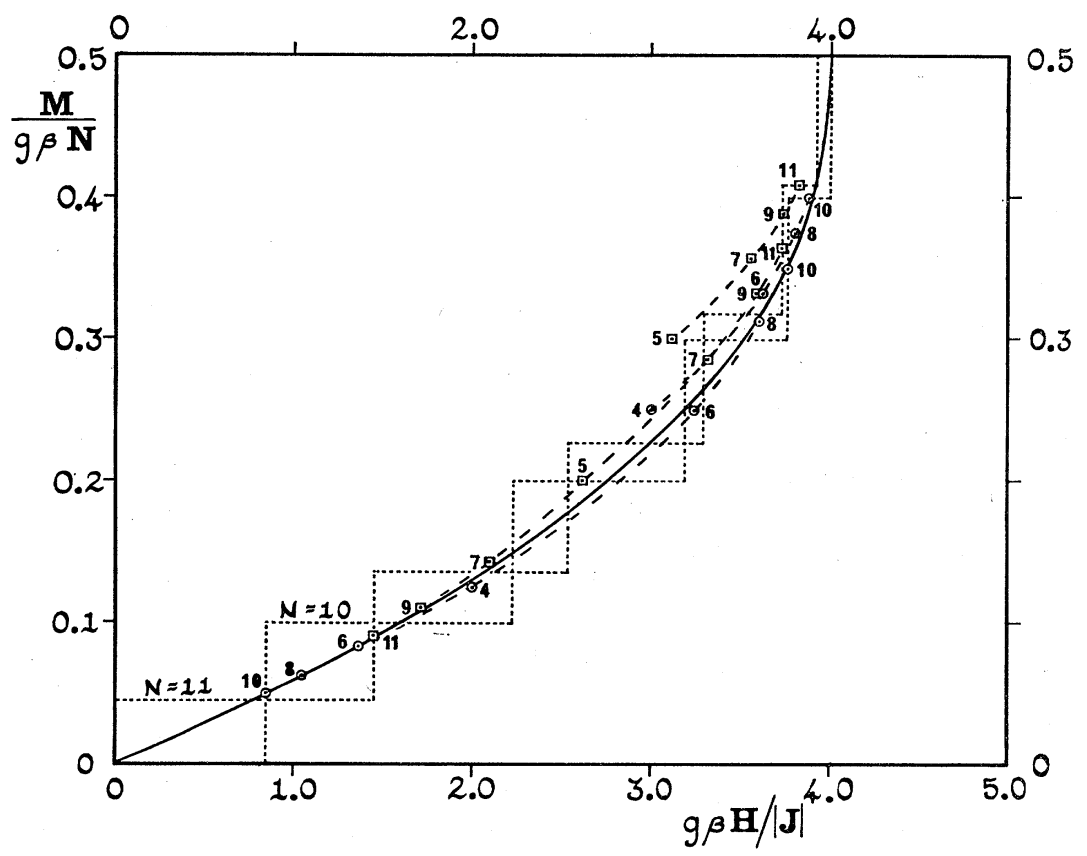

FIG. 18. Estimation of the limiting zero-temperature magnetization curve from the midpoints of the steps of the finite $N$ curves [circles $N$ even and squares $N$ odd]. The zero-point step functions corresponding to $N=10$ and 11 are shown dotted.

implies that the limiting susceptibility for $\gamma=1$ can approach a finite nonzero value $\chi_{0}$ at $T=0$.

To illustrate the detailed mechanism by which this occurs, we have sketched in Fig. 16 the relevant energy levels for (a) $\gamma<1$ and (b) $\gamma=1$ for finite systems ${ }^{39}$ and for the corresponding infinite systems as a function of magnetic field. The susceptibility at $T=0$ is essentially the curvature of the ground state $E_{0}(H)$ since $\chi(H)$ $=\left(\partial^{2} / \partial H^{2}\right) E_{0}(H)$. [Similarly the magnetization is just the slope of $\left.E_{0}(H).\right]$ For finite $N$, the ground state (marked by a bold curve) consists of a series of straight lines and consequently (for $N$ even) the curvature for small $H$ is always zero, so that $\chi_{N}(0) \equiv 0$ for all $\gamma$. In the limit $N \rightarrow \infty$, however, the ground-state curve for $\gamma=1$ is smooth with continuous slope and definite (in general nonzero) curvature. When $\gamma<1$, on the other hand, the limiting curve consists of a horizontal straight line out to a finite field strength $H_{a}$ at which the anisotropy gap vanishes. The curvature, and hence the susceptibility, near $H=0$ remains zero.

It is worth remarking that the existence of a nonzero $\chi_{0}$ for isotropic coupling can be demonstrated rigorously for chains in the limiting case of infinite spin. ${ }^{31}$

To estimate roughly the value of the limiting zeropoint susceptibility $\chi_{0}$ for $S=\frac{1}{2}$, one may examine the trend of the means of $\chi_{N}(T)$ for $N=9$ and 10, and $N=10$ and 11 in the range $k T /|J|=0.4$ to 0.8 (see Fig. 14). These curves suggest that $\chi_{0}$ lies between 0.045 and 0.06 (in units of $g^{2} \beta^{2} N /|J|$ ). Indeed, some time ago, Hulthén, by means of an approximate calculation, estimated that $\chi_{0} \simeq 0.0591 g^{2} \beta^{2} N /|J| .^{40}$ In an attempt

${ }^{39}$ Accurate graphs of all the energy levels as a function of field are given in Ref. 10 for $N=8$.

${ }_{40}$ Reference 7, p. 78. to improve this estimate, we examined the magnetization curve for the finite chains, which is also of interest in its own right.

Figure 17 shows the magnetization for a chain of $N=10$ spins as a function of field for different temperatures. For temperatures above $k T /|J|=0.3$ the curves are smooth and investigation of the convergence with $N$ suggests that the limiting curves are well approximated. For lower temperatures the magnetization displays oscillations and approaches a step function at $T=0$, the discontinuities being $\Delta M / M_{\max }=2 / N$. This behavior is, of course, just what follows from our previous discussion of the ground state when $N$ is finite.

Despite the discontinuities, one notices that the midpoints of the vertical and horizontal parts of the steps lie near a smooth curve which presumably approximates the limiting $(N=\infty)$ zero-temperature magnetization curve. This is confirmed by Fig. 18 where the midpoints for a number of the longer chains are plotted. The solid line shows the estimated limiting curve which should be accurate to within about $1 \%$ of the saturation value. This is supported by the analytic calculations of Griffiths. ${ }^{41}$

As mentioned previously, the magnetization at $T=0$ attains its saturation value at a finite critical field $H_{s}=4|J| / g \beta$. Below this critical field the magnetization appears to follow a square root law

$$
M / M_{\mathrm{max}}=1-A\left[1-\left(H / H_{s}\right)\right]^{1 / 2}
$$

as $H \rightarrow H_{s}$ with $A \approx 1.2-1.3 \approx 4 / \pi=1.2732$. (For further discussion, see below.) This behavior is also a

${ }^{41}$ R. B. Griffiths (private communication); Phys. Rev. 133, A768 (1964). 
feature of the transverse $\left(J^{\|}=0\right)$ model solved rigorously by Katsura. ${ }^{29}$ In that case $A=2 \sqrt{2} / \pi=0.9003$.

The slope of the limiting magnetization curve at $H=0$ is the zero-point susceptibility $\chi_{0}$. It seems likely that the midpoints of the finite $N$ magnetization steps approach the limiting curve as fast as $1 / N$. Consequently, if the limiting magnetization $M(H)$ has a Taylor series expansion in $H$ about $H=0$, one would expect the gradients $g_{1, N}=\frac{1}{2} \Delta M_{1, N} / \Delta H_{1, N}$ of the lines from the origin to the midpoints of the first steps (for $N$ even), and similar gradients to other steps, to approach the limiting slope $\chi_{0}$ as fast as $1 / N$. Examination of the gradients $g_{j, N}$ for small $j$ and $N=4$ to $11^{42}$ does yield roughly linear plots versus $1 / N$ which, if extrapolated, suggest that $\chi_{0} /\left(g^{2} \beta^{2} N /|J|\right)$ lies in the range $0.0555 \pm 0.0020$. This is significantly lower than Hulthén's estimate of 0.0591 .

This estimate rests on the assumption that the derivative of $M(H)$ does not vary too rapidly near $H=0$. Since this work was performed, however, Griffiths ${ }^{41}$ has produced strong (though not quite rigorous) theoretical arguments based on an analysis of the "unbound" antiferromagnetic spin-wave states which show (a) that $M(H)$ is nonanalytic at $H=0\left(\partial^{2} M / \partial H^{2}\right.$ diverging sharply to $+\infty$ as $H \rightarrow 0$ ) and, (b) that

$$
\chi_{0} /\left(g^{2} \beta^{2} N /|J|\right)=0.050661 \cdots \approx 1 / 2 \pi^{2} .
$$

In view of Walker's results ${ }^{9}$ mentioned in the Introduction, which show that the point $\gamma=1, T=0, H=0$ is in some ways analogous to a phase transition point, ${ }^{15}$ one should not really be surprised that $M(H)$ is nonanalytic at $H=0$. The divergence of $\partial^{2} M / \partial H^{2}$ shows why extrapolation of the gradients $g_{j, N}$ linearly with $1 / N$ leads to an overestimate of $\chi_{0}$. [Essentially $\chi(H)$ for small $H$, of order $1 / N$, is well approximated by the $g_{j, N}$ but does not itself approximate $\chi_{0}$ well unless $H$ is exponentially small.] It seems very probable that $1 / 2 \pi^{2}$ is the exact constant in (4.6) so that the estimate from the gradients is $10 \%$ high.

Accepting (4.6) as correct we may complete the estimation of the antiferromagnetic susceptibility for $\gamma=1$ down to zero temperature. The result is shown in Fig. 14 (as a dashed curve) and in Fig. 15. Above $k T /|J|=0.60$ it should be accurate to within $1 \%$ but in the region $k T /|J|=0.05$ to 0.50 the error might perhaps rise to $5 \%$.

The zero-temperature magnetization curve for other values of $\gamma$ may be studied as for $\gamma=1$. The critical field $H_{s}$ is determined by the intersection of the energy level for total $S^{z}=\frac{1}{2} N$ (a component of the zero-field ferromagnetic ground state) with the lowest level for $S^{z}=\frac{1}{2} N-1$ (single ferromagnetic spin wave) ${ }^{39}$ both of which are known exactly. Thus,

$$
H_{s}(\gamma)=2(1+\gamma)|J| / g \beta .
$$

${ }^{42}$ The gradients from the origin to the midpoints of the vertical parts of the steps are given by

$$
\begin{array}{ll}
g_{j, N}=\left(j-\frac{1}{2}\right) \Delta M_{j, N} / \Delta H_{j, N} & \text { for } N \text { even and } \\
g_{j, N}=j \Delta M_{j, N} / \Delta H_{j, N} & \text { for } N \text { odd. }
\end{array}
$$

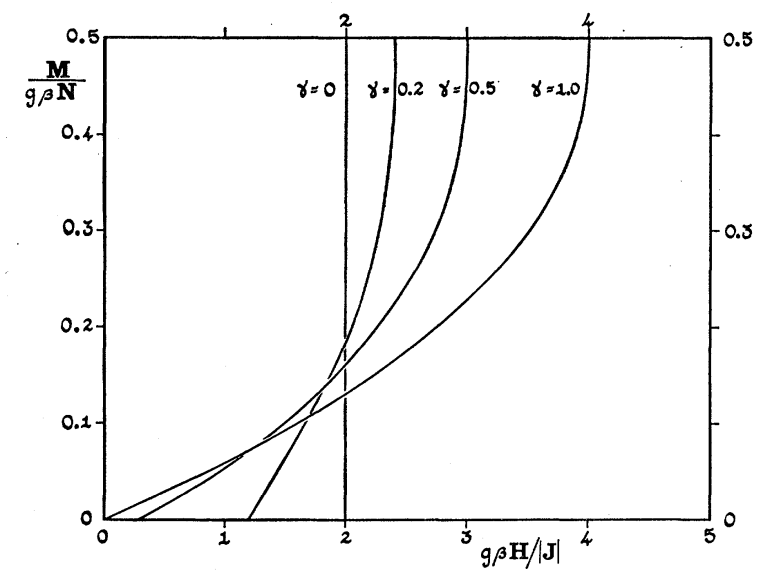

Frg. 19. Dependence of zero-temperature magnetization curves on anisotropy.

In order to study the form of the magnetization curve nęar saturation, it is necessary to consider the width of the first magnetization step away from saturation (see Fig. 18). The position $H=H_{1}$ of this step is given by the intersection of the lowest level for $S^{z}=\frac{1}{2} N-1$ with the lowest level for $S^{z}=\frac{1}{2} N-2$ which in the limiting case, $N \rightarrow \infty$, is given by $-4|J|(1+\gamma)$ [Eq. (5.4), Sec. 5 , with $k=2 \pi]$. It is seen, however, that use of the limiting curve again yields $H_{s}$; it appears, therefore, that the width of this step is directly determined by the energy discrepancy $\Delta E_{1}$ between the lowest lying level for $S^{z}=\frac{1}{2} N-2$ (finite $N$ ) and its limiting value. An estimate of the lowest, finite $N$, energy level as a function of $\gamma$ may be obtained, for example, by applying second-order perturbation theory to the appropriate submatrices of the Hamiltonian (1.1). One finds that for large $N, \Delta E_{1} \simeq 2 \gamma \pi^{2}|J| / N^{2}$. Corresponding to a magnetization step of $\Delta M=g \beta$ or $\Delta M / M_{\max }=2 / N$, we thus find a magnetic field step $\Delta H_{1}=\frac{1}{2}\left(H_{s}-H_{1}\right)$ $=\Delta E_{1} / 2 g \beta \simeq \gamma \pi^{2}|J| / g \beta N^{2}$. On eliminating $N$ we obtain

$$
\frac{\Delta M}{M_{\max }} \approx\left[\frac{8(1+\gamma)}{\pi^{2} \gamma}\right]^{1 / 2}\left(\frac{\Delta H_{1}}{H_{s}}\right)^{1 / 2},
$$

which suggests that when $N \rightarrow \infty$ we may write more generally, as $H \rightarrow H_{s}$,

with

$$
M / M_{\max }=1-A(\gamma)\left[1-\left(H / H_{s}\right)\right]^{1 / 2}
$$

$$
A(\gamma)=(4 / \pi)[(1+\gamma) / 2 \gamma]^{1 / 2} .
$$

This argument is, of course, not rigorous but it should be at least qualitatively correct and its accuracy is supported by the results for finite $N$ and by agreement with Griffiths' analysis at $\gamma=1 .{ }^{42}$

Figure 19 shows the complete estimated limiting magnetization curve for the Ising limit $\gamma=0$, where it is a simple step function, and for intermediate values of $\gamma$.

When $\gamma<1$ we must, as mentioned, distinguish between a perpendicular and a parallel susceptibility. 


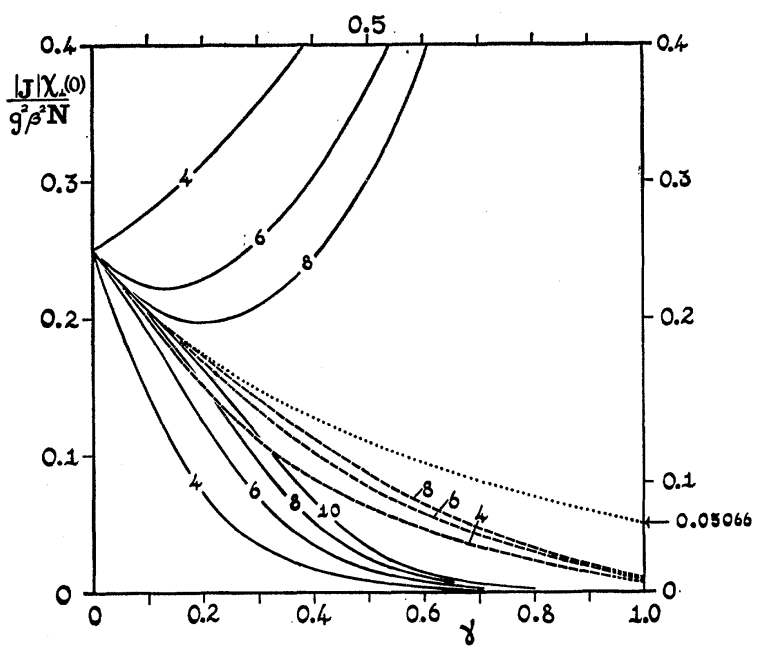

FIg. 20. Perpendicular susceptibilities for the ground and first $S^{z}=0$ excited states for finite rings (solid curves) and the geometric means (broken curves). The dotted curve represents the probable limit for $N \rightarrow \infty$.

For the Ising limit we have the exact result ${ }^{43,44}$

$$
\chi_{\perp}(T)=\frac{g^{2} \beta^{2} N}{4|J|}\left[\tanh K+K \operatorname{sech}^{2} K\right] \quad(\gamma=0),
$$

where $K$ is defined in (4.4). From this we see that $\chi_{\perp}(0)$ for $\gamma=0$, is nonzero. In the limit $N \rightarrow \infty$ this will remain true for all $\gamma$ and in fact as $\gamma \rightarrow 1, \chi_{\perp}(0, \gamma) \rightarrow \chi_{0}$. For this reason the susceptibility in the isotropic case is perhaps best regarded as a perpendicular susceptibility.

For finite $N$ the situation is again more complicated. The lowest set of solid curves in Fig. 20 show $\chi_{\perp N}(0, \gamma)$ for rings of $N=4,6,8$, and 10 spins. (The derivation requires the numerical calculation of matrix elements with the ground-state eigenvector.) As $\gamma \rightarrow 1$, for $N$ even and finite, $\chi_{\perp N}(0, \gamma)$ approaches zero as the previous arguments have shown it must. (For odd $N$ it diverges at $\gamma=1$.) Furthermore, for $\gamma>0.2$ the convergence for $N$ increasing is evidently very slow. The upper solid curves in Fig. 20 represent the perpendicular susceptibilities calculated for the lowest excited states. In the limit $N \rightarrow \infty$ these are expected to approach the groundstate results. The broken curves are the geometric means of these two sets of values and they seem to be converging somewhat more rapidly, at least for $\gamma<0.5$. Accepting the value (4.6) for $\chi_{0}$, the limiting zero-point perpendicular susceptibility $\chi_{\perp}(0, \gamma)$ must resemble in general form the dotted curve in Fig. 20. The slope of the limiting curve at $\gamma=0$ is known exactly, ${ }^{43}$ but for $0.2<\gamma$ $<0.5$ the dotted curve is probably accurate only to within $10 \%$.

\section{Ferromagnetic Coupling}

The susceptibility for ferromagnetic chains is conveniently discussed in terms of the deviations from

${ }^{43}$ M. E. Fisher, Physica 26, 618 (1960).

${ }^{44}$ M. E. Fisher, J. Math. Phys. 4, 124 (1963), and Ref. 29.
Curie's law. Quite generally we can write for the parallel susceptibility

where

$$
\chi_{N}(T)=\left[g^{2} \beta^{2} N / 4 k T\right] \xi_{N}(T),
$$

$$
\xi_{N}(T)=(4 / N)\left\langle\left[\sum_{i=1}^{N} S_{i}\right]^{2}\right\rangle
$$

the angular brackets denoting the canonical average. At high temperatures $\xi_{N}(T)$ approaches unity as $1 / T$. As the temperature falls $\xi_{N}(T)$ rises monotonically (for $J>0$ ) and, for finite $N$, levels off at a value determined by the properties of the ground state. For anisotropic chains $(\gamma<1)$ the ferromagnetic ground state is twofold degenerate with $\sum S_{i}{ }^{z}= \pm \frac{1}{2} N$ so that $\xi_{N}(0)=N$. In the isotropic $(\gamma=1)$ case, on the other hand, the ground state has total $\operatorname{spin} S=\frac{1}{2} N$ and hence is $(N+1)$-fold degenerate, $\sum S_{i}^{z}$ taking the values $\frac{1}{2} N$, $\frac{1}{2} N-1, \cdots-\frac{1}{2} N+1,-\frac{1}{2} N$. For finite pure Heisenberg chains, therefore, $\xi_{N}(T)$ rises to a maximum value $\xi_{N}(0)=\frac{1}{3}(N+2)$.

In the limit $N \rightarrow \infty$ we see that for all $\gamma, \xi(T)$ diverges as $T \rightarrow 0$. For Ising chains $(\gamma=0)$ this divergence is exponentially fast since we have rigorously

$$
\xi(T)=\exp [J / k T], \quad(\gamma=0) .
$$

This rapid divergence finds its origin in the anisotropy gap which ensures that all the pair correlation functions $\left\langle S_{i}{ }^{z} S_{j}{ }^{z}\right\rangle$ approach their zero-point values exponentially fast. For this reason we expect the limiting divergence for other values of $\gamma<1$ will also be of the form $\exp [\alpha J / k T]$ with $\alpha$ depending on the limiting anisotropy gap.

For pure Heisenberg coupling, however, a power law might be expected. Indeed, in the limit $S=\infty \quad(\gamma=1)$, the susceptibility diverges as $1 / T^{2}$ so that $\xi(T) \sim 1 / T$ as $T \rightarrow 0 .{ }^{31}$ Fig. 21 is a log-log plot of $\left[\xi_{N}(T)-1\right]$ versus the temperature for finite isotropic chains of $N=3,4$, $\cdots, 10,11$ spins. The curves evidently approach the

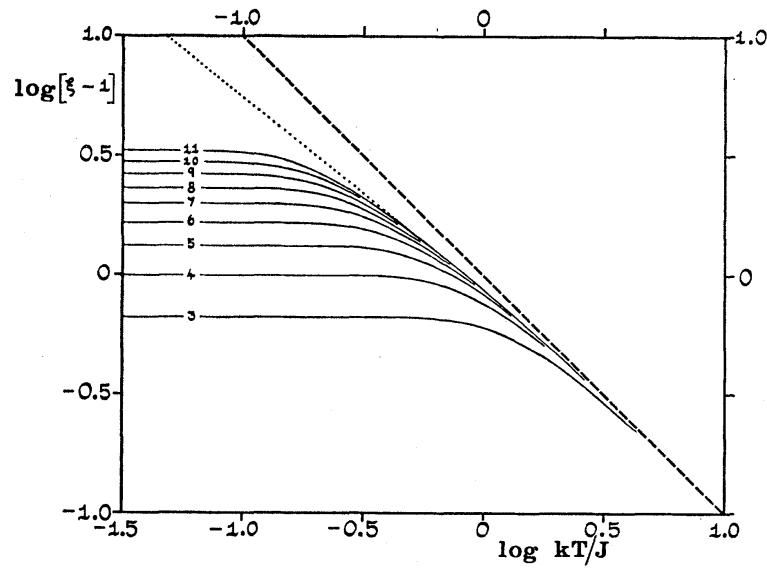

FIG. 21. Log-log plot of the reduced ferromagnetic susceptibility $\xi(T)-1$ for finite isotropic chains. 
limit monotonically from below. The convergence is quite rapid and for $k T / J>0.3$ the limiting curve is indicated quite accurately. At high temperatures the log-log plot becomes linear with slope unity as shown by the broken line in Fig. 21. This simply confirms the $1 / T$ deviations from Curie's law. At lower temperatures in the range $k T / J=0.25-1.0$, the limiting curve is again almost linear but with slope close to $\frac{4}{5}$ (see dotted line in Fig. 21). If the curves for larger $N$ continued this trend it would imply a divergence of $\xi(T)$ like $1 / T^{4 / 5}$ and of $\chi(T)$ like $1 / T^{9 / 5}$ as $T \rightarrow 0$. It is quite possible, however, that the true asymptotic behavior sets in only below $k T / J=0.2$. Nevertheless, the qualitative behavior is clearly rather similar to that for $S=\infty$.

The nature of the variation of the susceptibility with magnetic field can be seen in Fig. 22 which shows the ferromagnetic susceptibility of isotropic chains of $N=8$ spins in various fields. The convergence with $N$ is appreciably more rapid in a field than for $H=0$.

\section{SPECTRUM}

In the following account we confine our attention to a discussion of the spectrum of energy levels in the absence of an applied field, i.e., the eigenvalues of the Hamiltonian (1.1) with $H=0$.

\section{Ferromagnetic Coupling}

The ferromagnetic ground state for all $\gamma$ is, of course, exactly $E_{0}=-\frac{1}{2} N J$ corresponding to $\sum S_{i}{ }^{z}=\frac{1}{2} N$ (all spins aligned). With one "overturned spin" we have a

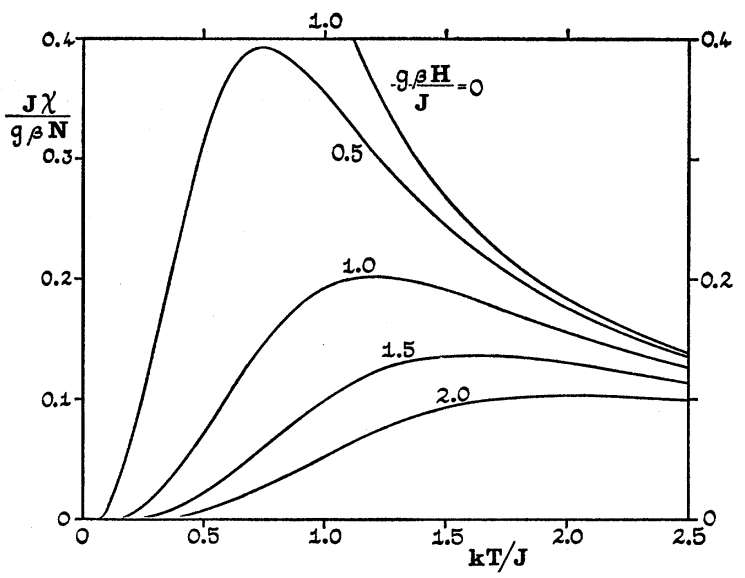

FIG. 22. Ferromagnetic susceptibility for rings of $N=8$ spins with isotropic coupling for different magnetic fields.

single "spin wave" of energy

where

$$
E-E_{0}=\epsilon(k)=2 J[1-\gamma \cos k],
$$

$$
k=2 \pi r / N, \quad r=0, \pm 1, \pm 2, \cdots .
$$

With two overturned spins, the simple spin-wave approximation predicts

$$
E-E_{0}=\epsilon\left(k_{1}\right)+\epsilon\left(k_{2}\right),
$$

but this is not exact owing to the spin-wave "interactions." For anisotropic linear chains the interaction of
FIG. 23. Energy levels for two overturned spins (interacting spin waves) for a ring of $N=11$ spins and $\gamma=1$. The arrows indicate the deviations from the levels for two independent spin waves. Note that we are taking an "antiferromagnetic view" of the energy levels $(J=-|J|)$, and each level has been normalized by subtraction of an energy $-\frac{1}{2} N J$.

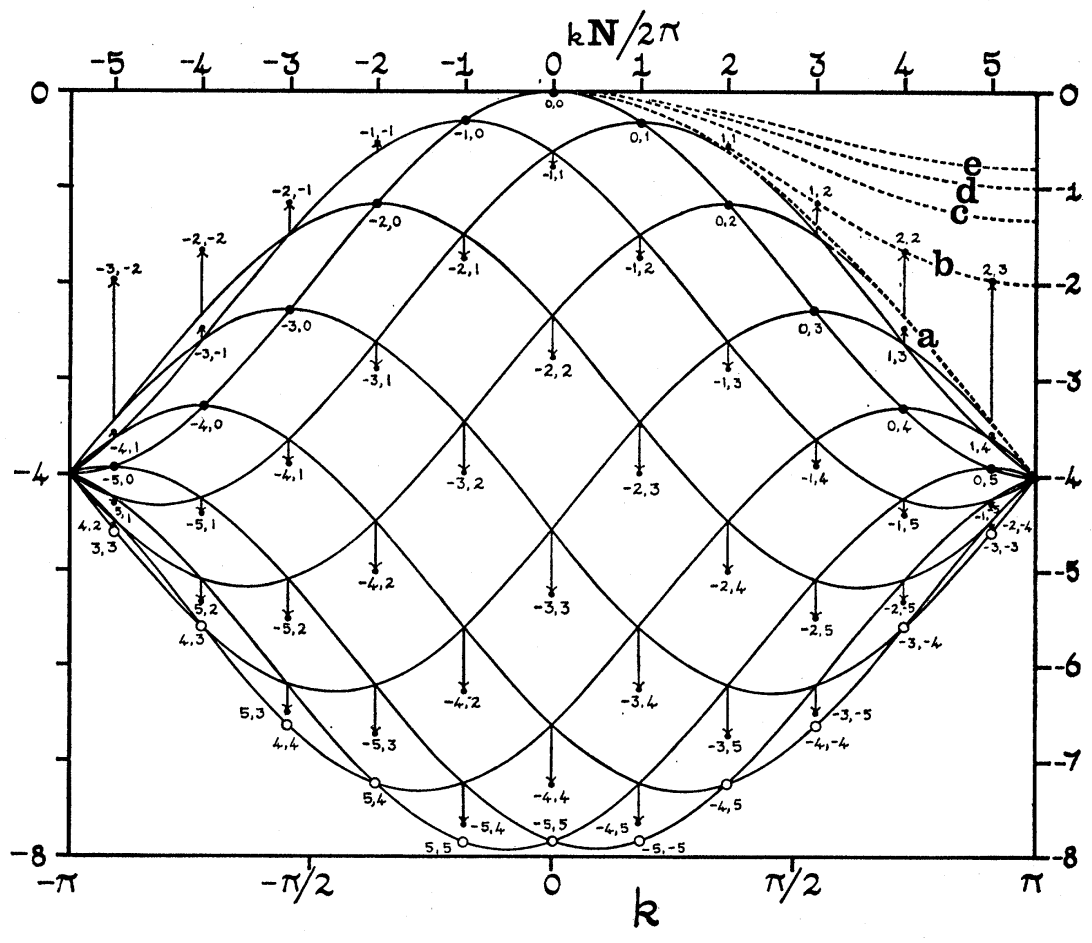




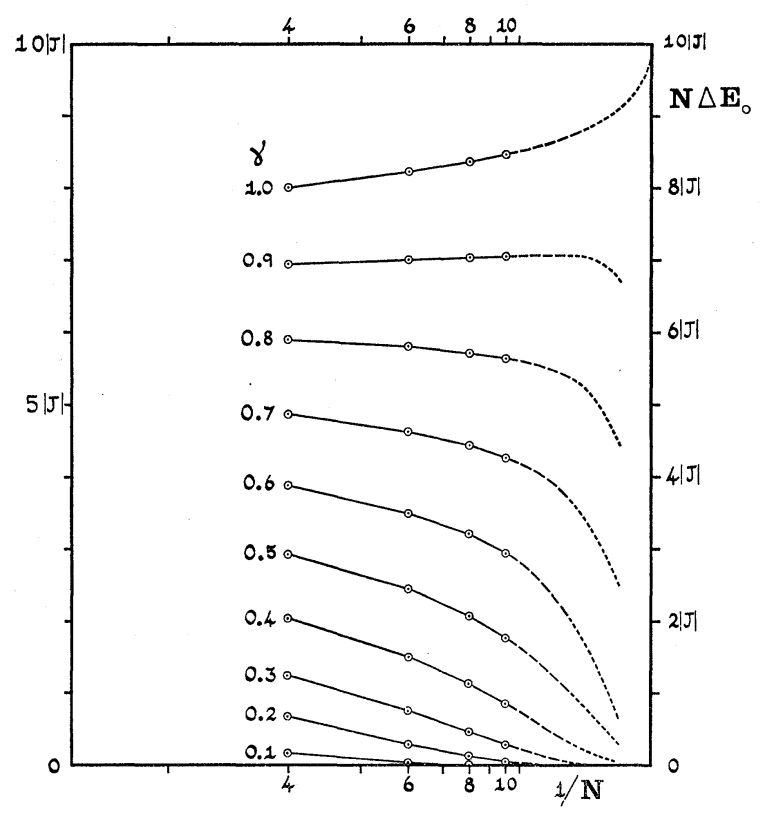

FIG. 24. Dependence of the splitting $\Delta E_{0}$ of the antiferromagnetic ground state on $\gamma$ and $N$.

two spin waves has been analyzed theoretically by Orbach, ${ }^{8}$ who followed Bethe's analysis for $\gamma=1 .^{6}$

A graphic understanding of these results can be obtained from Fig. 23, which shows energy levels for a ring of $N=11$ spins for $\gamma=1$. The solid curve through the dark circles is the single spin-wave dispersion law (5.1). The other solid curves represent the superimpositions (5.2), and their intersections are thus the spinwave approximations to the levels for two overturned spins. The arrows indicate the energy shifts due to the interactions, the exact energy levels lying at the points of the arrowheads.

The open circles represent "missing states," i.e., levels predicted by (5.2) which do not occur for the true Hamiltonian (essentially because two spins cannot be overturned at the same site). For most combinations of $k_{1}$ and $k_{2}$ the interactions are evidently repulsive and the spin waves "scatter" and remain "unbound" (category I states ${ }^{8}$ ). However, for $k_{1} \simeq k_{2} \simeq \frac{1}{2} k$, the interactions are strongly attractive and the spin waves condense into a bound state (category II) given by, ${ }^{8}$

$$
\epsilon_{\mathrm{II}}(k)=2 J\left[1-\frac{1}{2} \gamma^{2}(1+\cos k)\right] \text {. }
$$

This is indicated by the dotted line (b) in Fig. 23. As the form of this dispersion relation suggests, the corresponding eigenfunction relates to two closely associated overturned spins traveling around the ring together. The dotted line (a) in Fig. 23 is the $N=\infty$ limit of the energies of the unbound states namely, ${ }^{8}$

$$
\epsilon_{\mathrm{I}}^{\min }(k)=4 J\left(1-\gamma \cos \frac{1}{2} k\right),
$$

which is just the envelope of the curves (5.2).

One may in a similar way study numerically the bound states for $r$ overturned spins. The lowest states seem to follow the relation

$$
\epsilon^{(r)}(k) \simeq(2 J / r)[1-\cos k]
$$

when $\gamma=1$, as illustrated in curves (c), (d), and (e) of Fig. 23, corresponding to 3,4 , and 5 overturned spins, respectively. For $\gamma \neq 1$ the amplitudes of the $\cos k$ term appear to decrease as $\gamma^{r}$ or more rapidly.

\section{Antiferromagnetic Coupling}

The approach of the antiferromagnetic ground-state energies for finite chains to the limiting value was discussed in Sec. 2. The question of the degeneracy of the ground state has not, however, been considered. When $N$ is even ${ }^{45}$ the antiferromagnetic ground state at the Ising limit $(\gamma=0)$ is twofold degenerate. This degeneracy, however, is split by the transverse terms in the Hamiltonian, although, as is well known, the splitting only arises in $N$ th-order perturbation theory. This suggests that in the limit $N \rightarrow \infty$, the ground state should again become degenerate, at least in some sense.

To investigate this point we may examine the groundstate splitting $\Delta E_{0}(N)$ as a function of $N$. If $\Delta E_{0}$ varies as $1 / N$ for $N$ large, the levels close up, but only at a rate characteristic of a continuum of levels in the limit of $N=\infty$. Conversely, if $\Delta E_{0}$ vanishes more rapidly than $1 / N$, e.g., as $1 / N^{2}$, then we may consider the levels as asymptotically degenerate even if they lie within a continuum. In Fig. 24, the product $N \Delta E_{0}(N)$ has been plotted versus $1 / N$ for $N=4,6,8$, and 10 , and for values of $\gamma$ in the range 0 to 1 . For $\gamma \leqslant 0.5, N \Delta E_{0}$ is rapidly decreasing, and there seems little doubt that the limit is zero. For $\gamma=0.6,0.7$, and 0.8 , the decrease is slower but the rate increases for larger $N$, and it seems probable that the limit is again zero, as suggested by the broken lines. (These lines are purely suggestive and are not to be taken as numerical extrapolations.) At $\gamma=0.9$ the values of $N \Delta E_{0}$ at first increase slightly with $N$, but for $N=8$ and 10 they are almost equal, and we believe that larger values of $N$ would again yield a product decreasing at first slowly, but eventually rapidly. At the limit $\gamma=1$, on the other hand, $N \Delta E_{0}$ seems to be rising steadily and approaching a definite limit at $1 / N=0$, consistent with a state lying in a continuum bounded by the ground state. (Consideration of the des Cloizeaux-Griffiths analysis ${ }^{26,41}$ suggests that this limit should be $\pi^{2}|J|$ ).

In summary, we feel that the evidence of Fig. 24 definitely suggests that for all $\gamma<1$ (i.e., anisotropic coupling) $\Delta E_{0}$ decreases more rapidly than $1 / N$ $\left(N \Delta E_{0} \rightarrow 0\right)$, so that the limiting ground state may be said to be (twofold) degenerate. In the isotropic limit, however, $\Delta E_{0}$ decreases only as $1 / N$ and the anti-

${ }^{45}$ When $N$ is odd, the Ising ground state is $2 N$-fold degenerate, but for $\gamma>0$, only a fourfold degeneracy corresponding to $\Sigma S_{j}{ }^{2}$ $= \pm \frac{1}{2}$ remains. We will not consider this case further. 
ferromagnetic ground state should be regarded as nondegenerate.

For an Ising chain the first excited states lie at an energy $\Delta E_{A}(0)=2|J|$ above the ground states, but at the Heisenberg limit the ground state is the limit of a continuum, and there is no anisotropy gap. (This may be seen explicitly from the theoretical calculation of the antiferromagnetic spin-wave states for $\gamma=1$ by des Cloizeaux and Pearson. ${ }^{26}$ ) To estimate the limiting anisotropy gap $\Delta E_{A}(\gamma)$ for intermediate values of $\gamma$, we have studied (a) the difference $\Delta E_{A}(\gamma, N)$ between the "lowest excited state" $E_{s}(\gamma, N)$ [actually two degenerate $\left(S^{z}= \pm 1\right)$ states which must be distinguished from the higher component of the split ground state] and the finite $N$ ground state $E_{0}(\gamma, N)$, and (b) the gap $\Delta E_{A}{ }^{\prime}(\gamma, N)$ between $E_{s}(\gamma, N)$ and the limiting ground state $N|J| \mathcal{E}_{0}(\gamma, \infty)$. At the Heisenberg limit $E_{s}(\gamma, N)$ becomes a continuum state and the fact that $\Delta E_{A}(1, N)$ and $\Delta E_{A}{ }^{\prime}(1, N)$ fall to zero like $1 / N$ may be checked from the values for small finite $N$. For other values of $\gamma$ also, linear extrapolation with $1 / N$ seems to be appropriate in estimating the limit. The two sets of values form two distinct sequences but, as one would expect, they tend to the same limiting value. In the case $\gamma=1$, the lowest excited state $E_{s}(1, N)$ becomes degenerate with the continuum state $\left(S^{z}=0\right)$ split off the degenerate $(\gamma=0)$ ground state, and hence $N \Delta E_{A}(1, N)=N \Delta E_{0}$ tends to a limit [which, as mentioned, is probably $\pi^{2}|J|$ although $N \Delta E_{A}{ }^{\prime}(1, N)$ appears to tend to a slightly different value]. Figure 25 shows the variation with $\gamma$ of the gap for finite $N$ and the estimated limit $\Delta E_{A}(\gamma)$, which is probably accurate to within $\pm 0.05 J$. The main feature of interest is the very slow increase in the gap as $(1-\gamma)$ increases from zero to 0.3 or 0.4 .

The problem of the antiferromagnetic spin-wave spectrum has recently been studied in detail by des Cloizeaux and Pearson, ${ }^{26}$ who computed numerically the lowest category I states for $\gamma=1$ and rings of $N=6$, 8,16 , and 48 spins. They showed analytically that the limiting dispersion law is

$$
\epsilon(k)=E^{(1)}(k)-E_{0}=|J| \pi|\sin k| .
$$

Extrapolation of the appropriate energy differences versus $1 / N$ for $N$ up to 11 yields the amplitude $|J| \pi$ to within 5 to $10 \%$, which confirms the validity of extrapolating the spectral properties.

Mattheiss ${ }^{25}$ has given a plot of all the energy levels versus $k$ for $N=6$ and 8 . The results for higher values of $N$ are more complex since there are more levels but they do not differ much qualitatively and so we do not present a figure. Mattheiss remarked that for even $N=2 m$, the antiferromagnetic ground state corresponded to $k=0$ when $m$ was even, but to $k=\pi$ when $m$ was odd. For odd $N=2 m+1$, we have found that the two degenerate ground states are at $k \simeq \pm \frac{1}{2} \pi$, and the state at $k=0$ or $\pi$ is the higher according as $m$ is odd or

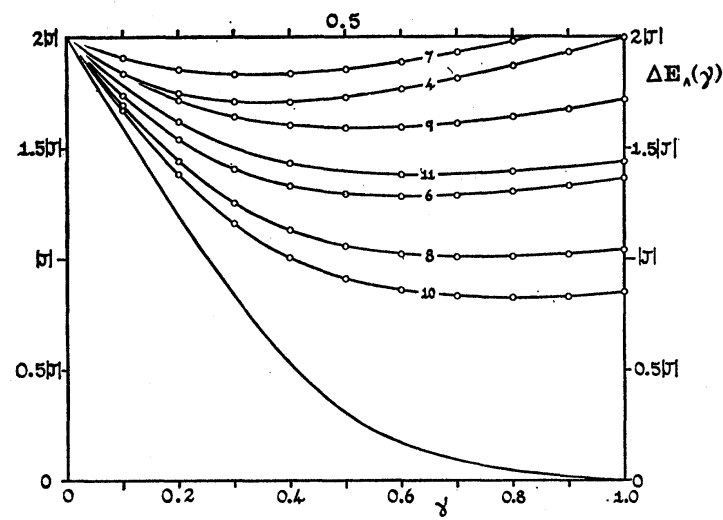

FIG. 25. Antiferromagnetic anisotropy gap $\Delta E_{A}(\gamma, N)$ and the estimated limit $\Delta E_{A}(\gamma)$.

even. ${ }^{46}$ As Mattheiss says, there seems to be no direct physical interpretation of these facts.

It would be of interest to decide how well the higher antiferromagnetic states can be represented as superimpositions of spin waves obeying (5.6). The situation seems appreciably more complex than for the ferromagnetic chains, and although the exact spectra do display levels that lie roughly on curves $|J| \pi\left(\left|\sin k_{1}\right|\right.$ $\left.+\left|\sin k_{2}\right|\right)$ with $k=k_{1}+k_{2}$, we have not been able to discover any simple numerical correlations, and some low-lying states (presumably "bound states") are definitely not representable in this way.

\section{ANTIFERROMAGNETIC CORRELATIONS AND ORDER}

For an antiferromagnetic chain at zero temperature the pair-correlation functions

$$
\omega_{l}(N)=4\left\langle S_{0}{ }^{z} S_{l}{ }^{z}\right\rangle=(4 / N) \sum_{j=1}^{N}\left\langle S_{j}{ }^{z} S_{j+l^{z}}\right\rangle
$$

can be calculated from the detailed expansion of the ground-state wave function in the basis of functions of definite "up" and "down" spins. Numerical results for $N=4,6,8$ and 10 , and $\gamma=0.1,0.3, \cdots, 1.0$ are given in Table II. For fixed $l$ the convergence in the case of $N$ even is not regular: for $\gamma<0.3$ it is monotone increasing and for $\gamma>0.8$ monotone decreasing, the curves for different $N$ crossing in the intermediate region. However, the convergence is quite rapid for $\gamma<0.3$ for all $l$, and for $l<\frac{1}{4} N$ in the case of $\gamma>0.8$. In the case of $N$ odd the convergence for $l<\frac{1}{2} N$ is monotone increasing for all $\gamma$ but rather slow. (These values are not tabulated.) The case of $l=\frac{1}{2} N$ is of special interest and will be discussed later.

Despite the relatively erratic convergence, extrapolation procedures for the limit $N \rightarrow \infty$ may be attempted and their accuracy checked against Orbach's exact calculation $^{8}$ of the limiting zero-temperature short-

\footnotetext{
${ }^{46}$ More precisely, the ground states are at $k= \pm \pi m / N$ for $m$ even and $k= \pm \pi(m+1) / N$ for $m$ odd.
} 
TABLE II. Antiferromagnetic ground state correlation functions $\omega_{l}(N, \gamma)$. [Note that $\omega_{0} \equiv 1$.]

\begin{tabular}{|c|c|c|c|c|c|c|c|}
\hline$N$ & $l$ & $\gamma=0.1$ & $\gamma=0.3$ & $\gamma=0.5$ & $\gamma=0.7$ & $\gamma=0.9$ & $\gamma=1.0$ \\
\hline 10 & $\begin{array}{l}1 \\
2 \\
3 \\
4 \\
5\end{array}$ & $\begin{array}{r}-0.99001 \\
0.98005 \\
-0.97998 \\
0.97988 \\
-0.97989\end{array}$ & $\begin{array}{r}-0.90791 \\
0.81790 \\
-0.81203 \\
0.80441 \\
-0.80475\end{array}$ & $\begin{array}{r}-0.77658 \\
0.56583 \\
-0.54351 \\
0.51181 \\
-0.51510\end{array}$ & $\begin{array}{r}-0.67994 \\
0.38839 \\
-0.35953 \\
0.31041 \\
-0.31865\end{array}$ & $\begin{array}{r}-0.62221 \\
0.28782 \\
-0.26199 \\
0.20558 \\
-0.21839\end{array}$ & $\begin{array}{r}-0.60206 \\
0.25407 \\
-0.23117 \\
0.17307 \\
-0.18781\end{array}$ \\
\hline 8 & $\begin{array}{l}1 \\
2 \\
3 \\
4\end{array}$ & $\begin{array}{r}-0.98972 \\
0.97948 \\
-0.97936 \\
0.97921\end{array}$ & $\begin{array}{r}-0.90139 \\
0.80498 \\
-0.79910 \\
0.79102\end{array}$ & $\begin{array}{r}-0.77381 \\
0.55947 \\
-0.54364 \\
0.51598\end{array}$ & $\begin{array}{r}-0.68355 \\
0.39250 \\
-0.37551 \\
0.33311\end{array}$ & $\begin{array}{r}-0.62819 \\
0.29464 \\
-0.28238 \\
0.23186\end{array}$ & $\begin{array}{r}-0.60852 \\
0.26104 \\
-0.25194 \\
0.19883\end{array}$ \\
\hline 6 & $\begin{array}{l}1 \\
2 \\
3\end{array}$ & $\begin{array}{r}-0.98821 \\
0.97645 \\
-0.97648\end{array}$ & $\begin{array}{r}-0.89102 \\
0.78410 \\
-0.78616\end{array}$ & $\begin{array}{r}-0.77359 \\
0.55640 \\
-0.56561\end{array}$ & $\begin{array}{r}-0.69291 \\
0.40440 \\
-0.42298\end{array}$ & $\begin{array}{r}-0.64155 \\
0.31068 \\
-0.33826\end{array}$ & $\begin{array}{r}-0.62284 \\
0.27735 \\
-0.30902\end{array}$ \\
\hline 4 & $\begin{array}{l}1 \\
2\end{array}$ & $\begin{array}{r}-0.98113 \\
0.96225\end{array}$ & $\begin{array}{r}-0.88125 \\
0.76249\end{array}$ & $\begin{array}{r}-0.78868 \\
0.57735\end{array}$ & $\begin{array}{r}-0.72542 \\
0.45084\end{array}$ & $\begin{array}{r}-0.68282 \\
0.36564\end{array}$ & $\begin{array}{r}-0.66667 \\
0.33333\end{array}$ \\
\hline
\end{tabular}

range order $\omega_{1}(\gamma)$. In Fig. 26 this exact result (a) is compared with an extrapolation estimate (b) based on sequences of means, $10 / 9,9 / 8,8 / 7$, etc., which show monotone increasing behavior for all $\gamma$. As may be seen, the agreement is very good (to within $1 \%$ ) and a similar technique has therefore been applied to estimate the limiting curve for the cases $\left|\omega_{2}(\gamma)\right|,\left|\omega_{3}(\gamma)\right|$, and $\left|\omega_{4}(\gamma)\right|$ (see Fig. 27). However, as the value of $l$ successively increases, the accuracy is expected to fall off somewhat, since there are fewer points to extrapolate.

Also shown in Fig. 26 are sums of Walker's perturbation series ${ }^{9}$

$$
\begin{aligned}
\omega_{1}=1-\gamma^{2}+\frac{3}{4} \gamma^{4}- & \left(7 / 2^{6}\right) \gamma^{8}-\left(9 / 2^{7}\right) \gamma^{10} \\
& -\left(11 / 2^{9}\right) \gamma^{12}+\left(13 / 2^{11}\right) \gamma^{14}+\cdots
\end{aligned}
$$

truncated at $\gamma^{14}$ [curve (c)] and at $\gamma^{6}$ [curve (d)]. In the former case the error is detectable only for $\gamma>0.85$ and reaches a maximum of only $6 \%$ at $\gamma=1$. In the latter case, however, the deviations are significant for $\gamma \geqslant 0.7$. Curve (e) is derived from Davis's perturbation

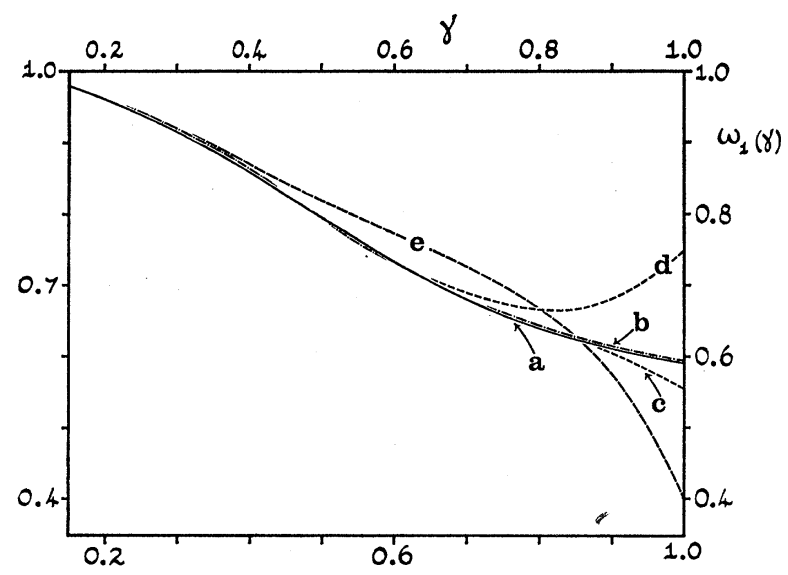

FIG. 26. Comparison of (a) the exact value of $\omega_{1}(\gamma)$ with (b) present extrapolations, (c) Walker's perturbation series to $\gamma^{14}$, (d) to $\gamma^{6}$ and (e) Davis's perturbation series. expansion $^{17}$ and is evidently less accurate than the extrapolations once $\gamma>0.40$ and seriously in error for $\gamma>0.85$.

An appreciation of the decay of the correlations with distance $l$ (as well as of the convergence in $N$ ) can be gained from Fig. $28_{\dot{0}}^{\nabla}$ which shows the finite ring correlations for $\gamma=0.3,0.5$, and 1.0. An alternating effect is evident, the values of $\left|\omega_{l}\right|$ for even $l$ being lower relative to those for odd $l$ than might be expected. For $l>\frac{1}{2} N$ the correlations, of course, start increasing as the points 0 and $l$ approach one another around the closed ring. Nevertheless, it is clear for $\gamma=0.3$, and reasonably so for $\gamma=0.5$, that the correlations for $l<\frac{1}{2} N$ are decaying to a constant level of about 0.8 and 0.5 , respectively. These values may be identified with the long-range order $\omega_{\infty}(\gamma)$ defined by

$$
\omega_{\infty}(\gamma)=\lim _{l \rightarrow \infty}\left|\lim _{N \rightarrow \infty} \omega_{l}(\gamma, N)\right|
$$

To estimate $\omega_{\infty}(\gamma)$ we have formed the minimum

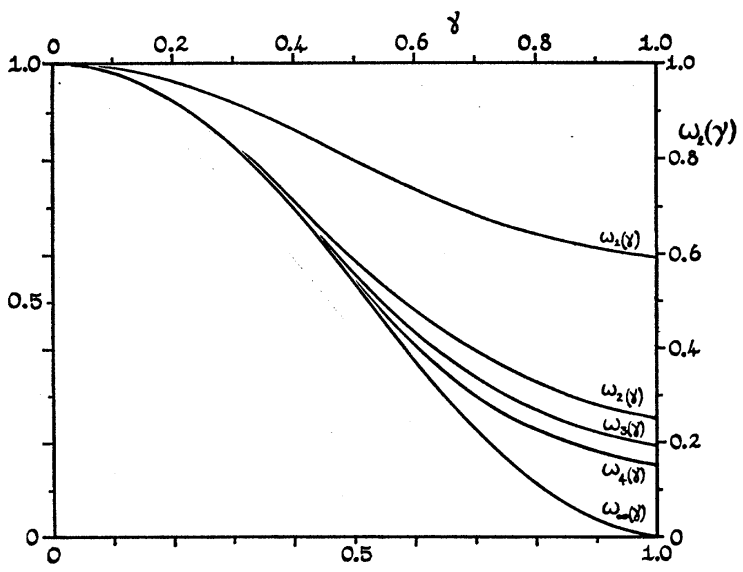

FIg. 27. Variation of the pair correlations $\left|\omega_{l}\right|$ with $\gamma$ for $l=1$ (exact) and $l=2,3,4$ (estimated) and $l=\infty$, the final estimate of the long-range order, 
means for $N$ even

$$
\omega_{\min }(\gamma, N)=\frac{1}{2}\left|\left[\omega_{\frac{1}{2} N}(\gamma, N)-\omega_{\frac{1}{2} N-1}(\gamma, N)\right]\right|
$$

and attempted to extrapolate to the limit $N=\infty$, which should agree with (6.3). These means are taken to reduce the alternation effects.

In this case we felt it was not advisable to use the results for odd $N$ in forming the estimate because, with the above definition of long-range order, interference effects around the chain occur near the Ising limit. (Note that for $N$ odd and $\gamma=0, \omega_{l}(N)=1-2 l / N, 0 \leqslant l<\frac{1}{2} N$.) For this reason we do not expect the limiting result for $\omega_{\infty}$ to have accuracy comparable with our estimate for $\omega_{1}$. (An estimate for $\omega_{1}$ using results for even $N$ only gives a result accurate to about $2.5 \%$.) Figure 29 shows a plot of $\omega_{\min }(N)$ versus $1 / N$. The points for $N>4$ appear reasonably collinear but care must be taken in extrapolation since curvature is to be expected for $\gamma<0.7$. (A similar, though less marked, effect occurs in the case of the short-range order.) For $\gamma<0.3$, the over-all range of variation is slight and an estimate can be made with some confidence: The limiting values here must, in fact, lie very close to the values for $N=10$. For $\gamma>0.7$ a linear extrapolation would seem to be reliable. For intermediate $\gamma$ some attempt has been made to allow for curvature effects but we would not claim very high accuracy for our results in this range. In fact, for $0.4<\gamma<0.6$, we have probably underestimated by several percent. We feel justified in concluding, however, that the long-range order vanishes only at the Heisenberg limit $\gamma=1$. In view of our conclusions regarding the asymptotic degeneracy of the ground state (and the vanishing of the anisotropy gap at $\gamma=1$ ) this is perhaps not unexpected.

To compare our estimate of the long-range order with other approximations we must recognize that most authors have used as an order parameter the so-called

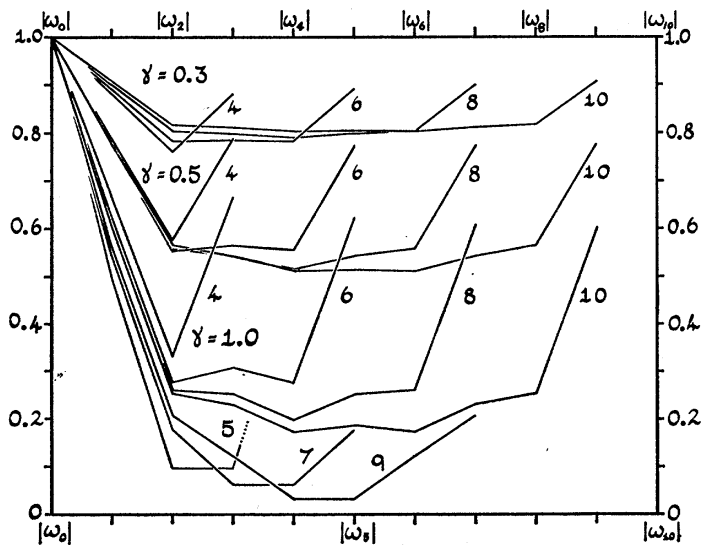

FIG. 28. Variation of $\left|\omega_{l}(N)\right|$ with $l$ for $\gamma=0.3,0.5$, and 1.0. and rings of $N=4,6,8$, and 10 spins.

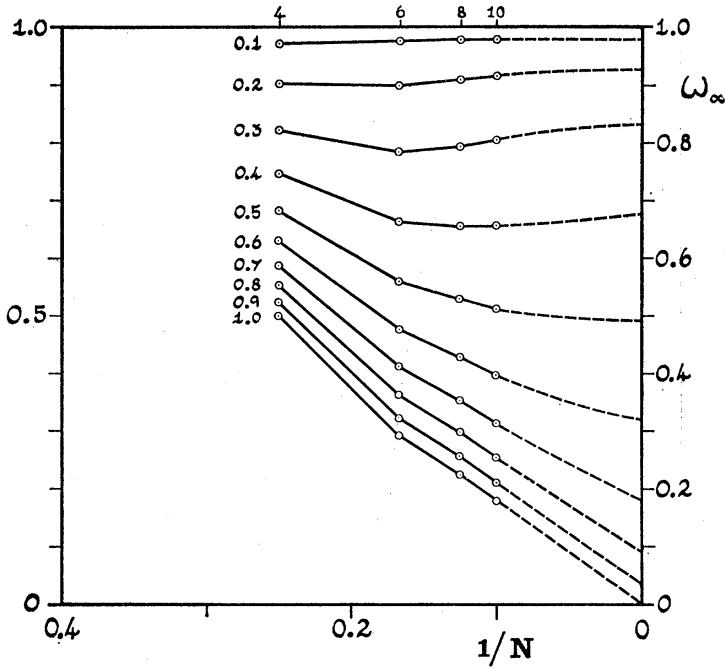

FIG. 29. Plot of $\omega_{\min }(\gamma, N)$ versus $1 / N$. Extrapolation yields an estimate of the long-range order.

"sublattice magnetization"9,14,17,47

$$
\sigma=\left(2 / \frac{1}{2} N\right)\left\langle\sum_{j \text { even }} S_{j}{ }^{2}\right\rangle=2\left\langle S_{0}^{z}\right\rangle
$$

where the angular brackets denote the canonical average. If this formula is interpreted literally, it is easily shown (on the grounds of spin reversal symmetry) that $\sigma$ always vanishes identically. Although this seems to have given rise to some confusion in the literature, ${ }^{20,48,49}$ the situation really parallels that in the ferromagnetic case which is quite well understood. ${ }^{50}$ If the (reduced) spontaneous magnetization of a ferromagnet is defined simply by $M_{0}=(2 / N)\left\langle\sum_{i} S_{i}{ }^{2}\right\rangle$, it also vanishes identically. The correct definition is made with the aid of a nonzero magnetic field, namely,

$$
M_{0}(T)=\lim _{H \rightarrow 0+} \lim _{N \rightarrow \infty}(1 / N) M_{N}(T, H),
$$

where

$$
M_{N}(T, H)=2 \sum_{j=1}^{N}\left\langle S_{j}{ }^{z}\right\rangle_{N}=2 N\left\langle S_{0}{ }^{z}\right\rangle_{N} .
$$

By considering the finiteness of the susceptibility per spin in the limit $H \rightarrow 0$ [which implies the convergence of the sum $\left.\sum_{l}\left(\left\langle S_{0}{ }^{z} S_{l}{ }^{z}\right\rangle-\left\langle S_{0}^{z}\right\rangle^{2}\right)\right]$ one can then see that

$$
\begin{aligned}
M_{0}^{2}(T) & =\lim _{N \rightarrow \infty}(1 / N)^{2} \sum_{i, j=1}^{N}\left\langle S_{i}{ }^{z} S_{j}{ }^{2}\right\rangle, \\
& =\lim _{l \rightarrow \infty} \lim _{N \rightarrow \infty}\left\langle S_{0}{ }^{z} S_{l}\right\rangle=\omega_{\infty}(T),
\end{aligned}
$$

${ }^{47}$ W. Marshall, Proc. Roy. Soc. (London) A232, 48 (1955).

${ }^{48}$ G. W. Pratt, Jr., Phys. Rev. 122, 489 (1961).

${ }^{49}$ N. Karayianis, C. A. Morrison, and D. E. Wortman, Phys. Rev. 126, 1443 (1962).

${ }^{50}$ See, for example, the discussion of the Ising model by G. F. Newell and E. W. Montroll, Rev. Mod. Phys. 25, 353 (1953). 


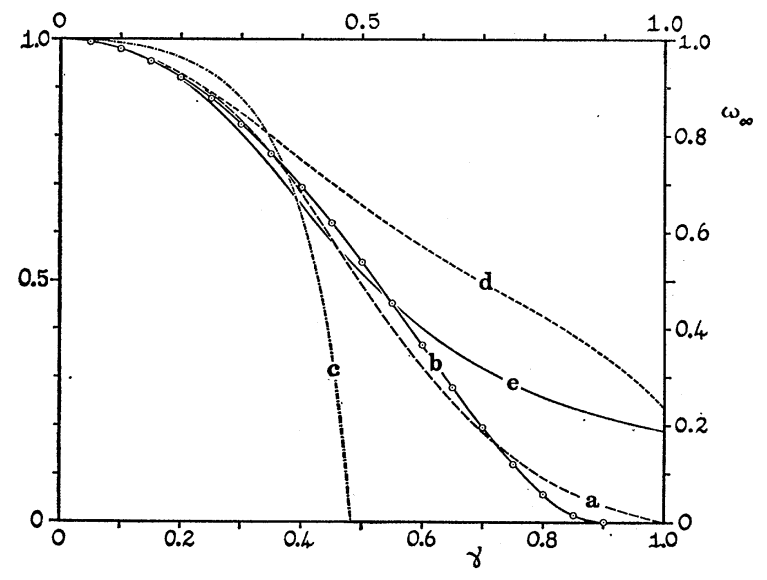

FIG. 30. Comparison of different approximations for the longrange order $\omega_{\infty}(\gamma)$ : (a) extrapolation estimates, (b) Walker's perturbation series to $\gamma^{6}$, (c) Kasteleyn's variational formula, (d) Davis's perturbation series, (e) $\omega_{\min }$ for a finite chain of 10 spins.

i.e., the long-range order is just equal to the square of the spontaneous magnetization.

For an antiferromagnetic one must introduce a "staggered" magnetic field $H^{*}$ by adding to the Hamiltonian a term

$$
\mathfrak{H}^{*}=H^{*} \sum_{j=0}^{N-1}(-)^{j} S_{j} z .
$$

With the aid of the corresponding "staggered magnetization" the sublattice magnetization may be defined properly by

$$
\begin{aligned}
\sigma & =\lim _{H^{*} \rightarrow 0+} \lim _{N \rightarrow \infty}(1 / N) M_{N}{ }^{*}\left(H^{*}\right), \\
& =\lim _{H^{*} \rightarrow 0+}\left[\left\langle S_{0}^{z}\right\rangle_{\infty}{ }^{*}-\left\langle S_{1}\right\rangle_{\infty}{ }^{*}\right] .
\end{aligned}
$$

In a similar way one can then conclude that the long-range order defined by (6.3) is related to $\sigma$ by $^{51}$

$$
\omega_{\infty}=\sigma^{2} \text {. }
$$

We"thus see that the two-order parameters are equivalent. One may, of course, avoid the somewhat artificial introduction of a staggered field by sticking to the definition (6.3) in terms of the correlation functions which, as argued by Ruijgrok and Rodriguez, ${ }^{20}$ also has other features to recommend it. In practice, however, it is often easier to compute $\sigma$ (or $M_{0}$ ) than to compute $\omega_{\infty}$ directly.

With these preliminaries we may compare our estimate of $\omega_{\infty}(\gamma)$ [curve (a) in Fig. 30] with Walker's exact perturbation expansion

$$
\sigma(\gamma)=1-\gamma^{2}-\frac{1}{4} \gamma^{4}-\frac{1}{16} \gamma^{6}+\cdots
$$

[curve (b),$^{9}$ with Kasteleyn's variational formula [curve (c) $],{ }^{14}$ and with Davis's perturbation formula

${ }^{51}$ Strictly, it may be necessary to define the long-range order as an average over the two sublattices [e.g., if the limit in (6.3) differs as $l \rightarrow \infty$ through even or through odd integers]. [curve (d)].17 Also shown in Fig. 30 are the values $\omega_{\min }(10)$ [curve (e)]. Up to $\gamma=0.3$, all approximations agree well with the exact series but at this point Davis's approximation (d) starts deviating seriously and predicts a relatively large nonzero value for $\omega_{\infty}(1)$. Kasteleyn's approximation (c) falls away sharply to zero at $\gamma_{c}=0.483$, which is surely incorrect. Our estimated curve falls some $10 \%$ below the series value in the region $\gamma=0.45$ to 0.65 , although the trend is very similar. In this region the series is probably still converging rapidly, as suggested both by the numerical magnitude of the terms, and by the agreement of the exact short-range order with the corresponding series up to $\gamma^{6}$ (Fig. 26). Above $\gamma=0.7$ we must expect the series to deviate from the true value and indeed the series for $\omega(\gamma)$ (rather than for $[\sigma(\gamma)]^{2}$ ) then yields lower values and has its zero at $\gamma=0.817$ rather than at $\gamma=0.897$. As already observed our extrapolated $\omega_{\infty}(\gamma)$ does not vanish until $\gamma=1$. Although we suspect this is the true situation it would be of great interest to have more precise information-ideally the rigorous answer -in the range above $\gamma=0.8$ ! At present it seems fair to conclude that the exact series expansion provides the best approximation up to $\gamma=0.75$ and that the true long-range order is unlikely to vanish for $\gamma<0.85$ and probably vanishes at $\gamma=1$.

In Fig. 27, therefore, we have plotted together with $\omega_{1}(\gamma)$ [exact] and our results for $\left|\omega_{2}\right|,\left|\omega_{3}\right|$, and $\left|\omega_{4}\right|$, what we think is the best estimate so far for $\omega_{\infty}(\gamma)$. This curve follows the series expansion up to $\gamma=0.6$, and thereafter has the same form as our extrapolation curve (a) in Fig. 30, approaching the latter from above and in close agreement for $\gamma>0.8$.

The excellent convergence of Walker's perturbation series in $\gamma$ suggests that this approach should be developed further for two- and three-dimensional lattices where convergence seems to be even better. We remark in passing that it is possible to calculate exactly the $\gamma^{2}$ correction to the energy and specific heat for all temperatures in the case of two-dimensional lattices for which the Ising problem has been solved, by expressing the perturbation in terms of the multiple correlation functions for $\gamma=0 .{ }^{52}$

\section{ACKNOWLEDGMENTS}

Weare grateful for the interest of Professor C. Do m b and Dr. M. F. Sykes and for the assistance of members of the University of London Computer Unit. One of us (J.C.B.) is indebted to the Department of Scientific and Industrial Research for the award of a maintenance grant. As mentioned in the Introduction, we have greatly benefited from correspondence and discussion with Dr. R. L. Orbach and Dr. R. B. Griffiths, who also made their own computations freely available to us. We wish to apologize to Dr. Griffiths for inadvertently delaying publication of his own work.

\footnotetext{
${ }^{52}$ The technique is similar to that expounded in Ref. 43.
} 